\title{
Octa- and Nonamethylfluorene and an Electron-Rich Permethylfluorenyl Ruthenocene Derivative
}

\author{
Patrick Bazinet, Karl A. Tupper, and T. Don Tilley* \\ Department of Chemistry, University of California at Berkeley, Berkeley, CA 94720-1460, USA \\ Chemical Sciences Division, Ernest Orlando Lawrence Berkeley National Laboratory, One Cyclotron \\ Road, Berkeley, CA 94720-1460, USA
}

\section{Supporting Information}

Crystallographic data (tables and CIF files) for 1, 2, and $\mathbf{4}$ : 


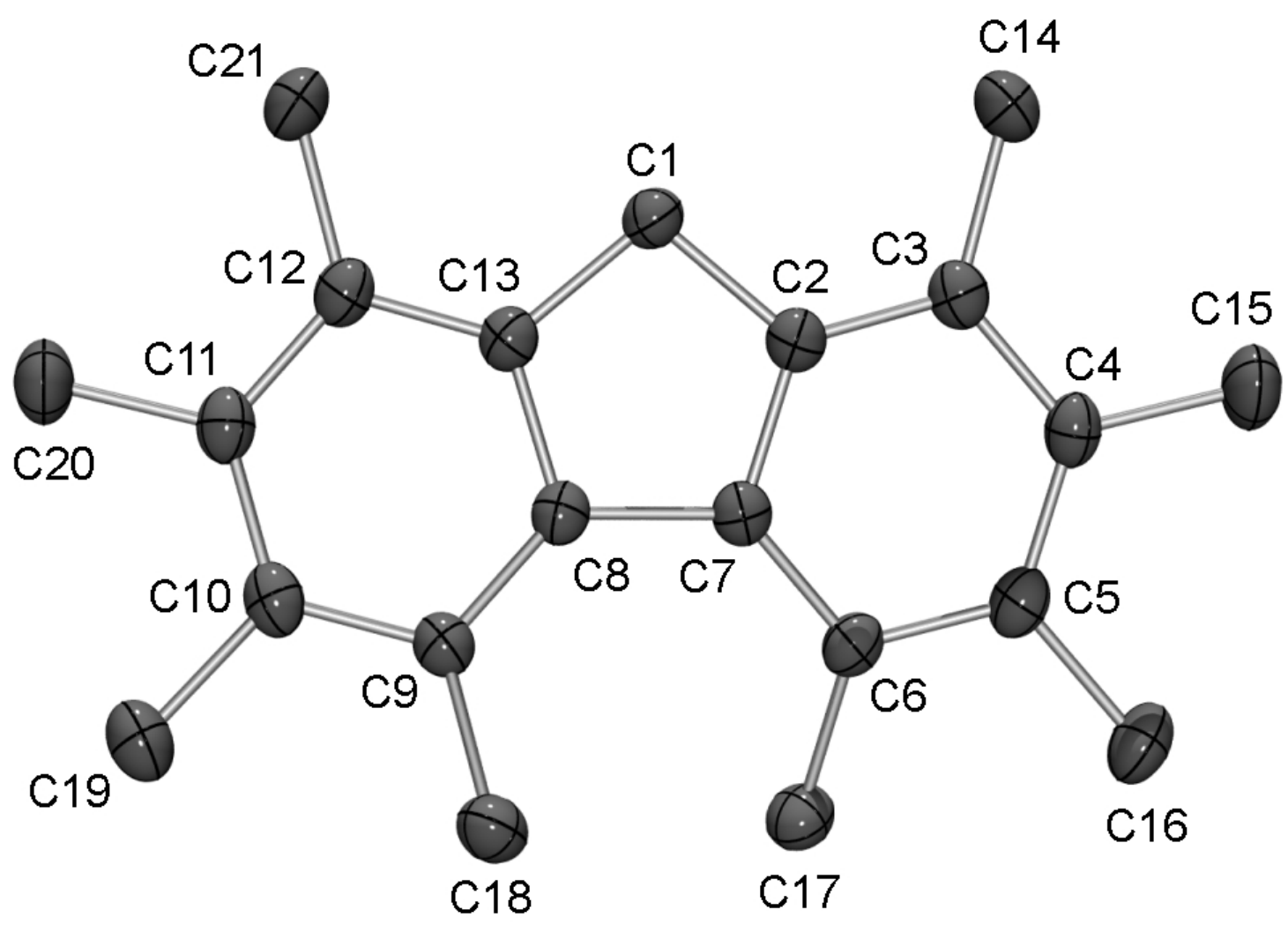

Figure 1. Thermal ellipsoid plot for compound $\mathrm{C}_{13} \mathrm{Me}_{8} \mathrm{H}_{2}$ (1). Hydrogen atoms are omitted for clarity. Thermal ellipsoids are drawn at $50 \%$ probability. 


\section{A. Crystal Data}

Empirical Formula

Formula Weight

Crystal Color, Habit

Crystal Dimensions

Crystal System

Lattice Type

Lattice Parameters
$\mathrm{C}_{21} \mathrm{H}_{26}$

278.42

off white, blades

$0.22 \times 0.37 \times 0.06 \mathrm{~mm}$

orthorhombic

Primitive

$$
\begin{aligned}
& a=17.4698(8) \AA \\
& b=24.6082(11) \AA \\
& c=7.3072(3) \AA \\
& \mathrm{V}=3141.4(2) \AA^{3}
\end{aligned}
$$

Space Group

$\mathrm{Z}$ value

Pccn (\#56)

8

$1.177 \mathrm{~g} / \mathrm{cm}^{3}$

$\mathrm{F}_{000}$

$\mu(\operatorname{MoK} \alpha)$
1216

$0.066 \mathrm{~mm}^{-1}$ 


\section{B. Intensity Measurements}

Diffractometer

Radiation

Detector Position

Exposure Time

Scan Type

$2 \theta_{\max }$

No. of Reflections Measured

Corrections

\section{Bruker APEX CCD}

$\operatorname{MoK} \alpha(\lambda=0.71073 \AA)$

graphite monochromated

$60.00 \mathrm{~mm}$

10.0 seconds per frame.

$\omega$ (0.3 degrees per frame)

$49.4^{\circ}$

Total: 14869

Unique: $2679\left(\mathrm{R}_{\mathrm{int}}=0.037\right)$

Lorentz-polarization

Absorption $\left(\mathrm{T}_{\max }=1.00 \mathrm{~T}_{\min }=0.92\right)$ 


\section{Structure Solution and Refinement}

Structure Solution

Refinement

Function Minimized

Least Squares Weights

p-factor

Anomalous Dispersion

No. Observations (I>-10.00б(I))

No. Variables

Reflection/Parameter Ratio

Residuals: R; Rw; Rall

Goodness of Fit Indicator

Max Shift/Error in Final Cycle

Maximum peak in Final Diff. Map

Minimum peak in Final Diff. Map
Direct Methods (SIR97)

Full-matrix least-squares

$\Sigma \mathrm{w}(|\mathrm{Fo}|-|\mathrm{Fc}|)^{2}$

$1 / \sigma^{2}(\mathrm{Fo})=4 \mathrm{Fo}^{2} / \sigma^{2}\left(\mathrm{Fo}^{2}\right)$

0.0600

All non-hydrogen atoms

2679

294

9.11

$0.0387 ; 0.0955 ; 0.0524$

1.014

0.00

$0.21 \mathrm{e}^{-/ \AA^{3}}$

$-0.15 \mathrm{e}^{-/ \AA^{3}}$ 


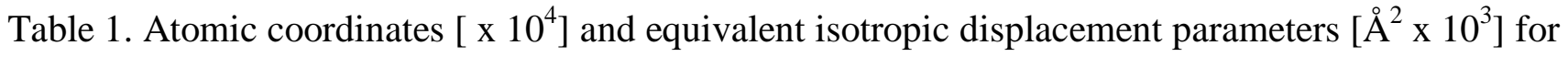
$\mathrm{Me}_{8} \mathrm{C}_{13} \mathrm{H}_{2}$. U(eq) is defined as one third of the trace of the orthogonalized $\mathrm{U}_{\mathrm{ij}}$ tensor.

\begin{tabular}{lcccc}
\hline & & & & \\
& $\mathrm{x}$ & $\mathrm{y}$ & $\mathrm{z}$ & $\mathrm{U}(\mathrm{eq})$ \\
\hline & & & & \\
$\mathrm{C}(1)$ & $-489(1)$ & $4268(1)$ & $2250(2)$ & $26(1)$ \\
$\mathrm{C}(2)$ & $-348(1)$ & $3666(1)$ & $2102(2)$ & $25(1)$ \\
$\mathrm{C}(3)$ & $-896(1)$ & $3258(1)$ & $1978(2)$ & $28(1)$ \\
$\mathrm{C}(4)$ & $-645(1)$ & $2716(1)$ & $1985(2)$ & $29(1)$ \\
$\mathrm{C}(5)$ & $131(1)$ & $2594(1)$ & $2269(2)$ & $28(1)$ \\
$\mathrm{C}(6)$ & $678(1)$ & $3012(1)$ & $2489(2)$ & $25(1)$ \\
$\mathrm{C}(7)$ & $440(1)$ & $3552(1)$ & $2236(2)$ & $24(1)$ \\
$\mathrm{C}(8)$ & $854(1)$ & $4084(1)$ & $2231(2)$ & $24(1)$ \\
$\mathrm{C}(9)$ & $1624(1)$ & $4223(1)$ & $1968(2)$ & $26(1)$ \\
$\mathrm{C}(10)$ & $1836(1)$ & $4774(1)$ & $2202(2)$ & $28(1)$ \\
$\mathrm{C}(11)$ & $1282(1)$ & $5180(1)$ & $2497(2)$ & $28(1)$ \\
$\mathrm{C}(12)$ & $497(1)$ & $5045(1)$ & $2499(2)$ & $27(1)$ \\
$\mathrm{C}(13)$ & $305(1)$ & $4500(1)$ & $2379(2)$ & $25(1)$ \\
$\mathrm{C}(14)$ & $-1733(1)$ & $3404(1)$ & $1901(3)$ & $36(1)$ \\
$\mathrm{C}(15)$ & $-1218(1)$ & $2258(1)$ & $1804(3)$ & $39(1)$ \\
$\mathrm{C}(16)$ & $368(1)$ & $2006(1)$ & $2391(2)$ & $35(1)$ \\
$\mathrm{C}(17)$ & $1462(1)$ & $2871(1)$ & $3235(2)$ & $32(1)$ \\
$\mathrm{C}(18)$ & $2207(1)$ & $3828(1)$ & $1208(2)$ & $33(1)$ \\
$\mathrm{C}(19)$ & $2671(1)$ & $4923(1)$ & $2142(3)$ & $38(1)$ \\
$\mathrm{C}(20)$ & $1521(1)$ & $5766(1)$ & $2710(3)$ & $40(1)$ \\
$\mathrm{C}(21)$ & $-125(1)$ & $5470(1)$ & $2621(3)$ & $35(1)$ \\
& & & & \\
\hline
\end{tabular}


Table 2. Hydrogen coordinates [ $\mathrm{x} 10^{4]}$ and isotropic displacement parameters $\left[\AA^{2} \times 10^{3}\right]$ for $\mathrm{Me}_{8} \mathrm{C}_{13} \mathrm{H}_{2}$.

\begin{tabular}{|c|c|c|c|c|}
\hline & $\mathrm{x}$ & $\mathrm{y}$ & $\mathrm{z}$ & $\mathrm{U}(\mathrm{eq})$ \\
\hline $\mathrm{H}(1 \mathrm{~B})$ & $-758(8)$ & $4421(6)$ & $1190(20)$ & $36(4)$ \\
\hline $\mathrm{H}(1 \mathrm{~A})$ & $-784(8)$ & $4354(6)$ & $3320(20)$ & $30(4)$ \\
\hline $\mathrm{H}(14 \mathrm{~B})$ & $-2013(11)$ & $3258(9)$ & $2980(30)$ & $66(6)$ \\
\hline $\mathrm{H}(14 \mathrm{C})$ & $-1807(12)$ & 3801(10) & $1930(30)$ & $81(7)$ \\
\hline $\mathrm{H}(14 \mathrm{~A})$ & $-1983(10)$ & $3257(7)$ & $800(20)$ & $57(5)$ \\
\hline $\mathrm{H}(15 \mathrm{C})$ & $-1715(14)$ & $2406(10)$ & $1580(30)$ & $97(8)$ \\
\hline $\mathrm{H}(15 \mathrm{~B})$ & $-1226(11)$ & $2042(8)$ & $2890(30)$ & $61(6)$ \\
\hline $\mathrm{H}(15 \mathrm{~A})$ & $-1078(11)$ & 1990(8) & $790(30)$ & $73(6)$ \\
\hline $\mathrm{H}(16 \mathrm{C})$ & $944(12)$ & $1960(8)$ & $2410(20)$ & $62(6)$ \\
\hline $\mathrm{H}(16 \mathrm{~B})$ & 181(9) & $1800(7)$ & $1320(20)$ & $53(5)$ \\
\hline $\mathrm{H}(16 \mathrm{~A})$ & 169(9) & 1841(6) & $3480(20)$ & $45(5)$ \\
\hline $\mathrm{H}(17 \mathrm{C})$ & $1402(8)$ & $2578(6)$ & $4250(20)$ & $42(4)$ \\
\hline $\mathrm{H}(17 \mathrm{~B})$ & 1817(9) & $2725(6)$ & $2290(20)$ & $37(4)$ \\
\hline $\mathrm{H}(17 \mathrm{~A})$ & $1708(8)$ & $3205(6)$ & 3821(19) & $32(4)$ \\
\hline $\mathrm{H}(18 \mathrm{C})$ & $2521(8)$ & $4007(6)$ & $240(20)$ & $42(4)$ \\
\hline $\mathrm{H}(18 \mathrm{~B})$ & $2589(10)$ & $3671(7)$ & $2130(20)$ & $38(4)$ \\
\hline $\mathrm{H}(18 \mathrm{~A})$ & $1940(8)$ & $3497(6)$ & $615(19)$ & $38(4)$ \\
\hline $\mathrm{H}(19 \mathrm{C})$ & $3000(16)$ & $4637(12)$ & $2490(30)$ & $110(9)$ \\
\hline H(19B) & $2814(12)$ & $5183(9)$ & $2960(30)$ & $78(7)$ \\
\hline H(19A) & $2845(12)$ & $5034(9)$ & $1000(30)$ & $95(8)$ \\
\hline $\mathrm{H}(20 \mathrm{C})$ & 1862(13) & $5895(10)$ & $1690(30)$ & $93(7)$ \\
\hline $\mathrm{H}(20 \mathrm{~B})$ & 1839(10) & $5818(7)$ & $3780(30)$ & $63(6)$ \\
\hline $\mathrm{H}(20 \mathrm{~A})$ & 1099(16) & $6004(12)$ & $2780(30)$ & $111(10)$ \\
\hline $\mathrm{H}(21 \mathrm{C})$ & $-621(15)$ & $5336(10)$ & $2550(30)$ & $90(8)$ \\
\hline $\mathrm{H}(21 \mathrm{~B})$ & $-83(12)$ & $5736(9)$ & $1630(30)$ & $83(7)$ \\
\hline $\mathrm{H}(21 \mathrm{~A})$ & $-80(12)$ & $5674(9)$ & $3730(30)$ & $88(7)$ \\
\hline
\end{tabular}


Table 3. Anisotropic displacement parameters $\left[\AA^{2} \times 10^{3}\right]$ for $\mathrm{Me}_{8} \mathrm{C}_{13} \mathrm{H}_{2}$. The anisotropic displacement factor exponent takes the form: $-2 \pi^{2}\left[\left(h a^{*}\right)^{2} U_{11}+\ldots+2 h k a * b * U_{12}\right]$

\begin{tabular}{|c|c|c|c|c|c|c|}
\hline & U11 & U22 & U33 & U23 & U13 & U12 \\
\hline $\mathrm{C}(1)$ & $23(1)$ & $29(1)$ & $28(1)$ & 1(1) & 1(1) & $0(1)$ \\
\hline $\mathrm{C}(2)$ & $25(1)$ & $26(1)$ & $23(1)$ & $1(1)$ & 1(1) & $-1(1)$ \\
\hline$C(3)$ & $26(1)$ & $31(1)$ & $27(1)$ & $2(1)$ & 1(1) & $-4(1)$ \\
\hline$C(4)$ & $33(1)$ & $28(1)$ & $26(1)$ & 1(1) & $2(1)$ & $-6(1)$ \\
\hline$C(5)$ & $36(1)$ & $25(1)$ & $23(1)$ & $1(1)$ & $3(1)$ & $-1(1)$ \\
\hline$C(6)$ & $28(1)$ & $24(1)$ & $24(1)$ & $0(1)$ & 2(1) & 1(1) \\
\hline$C(7)$ & $25(1)$ & $26(1)$ & $21(1)$ & $-1(1)$ & $2(1)$ & $-2(1)$ \\
\hline $\mathrm{C}(8)$ & $27(1)$ & $25(1)$ & $20(1)$ & $1(1)$ & $-1(1)$ & $0(1)$ \\
\hline $\mathrm{C}(9)$ & $24(1)$ & $31(1)$ & $23(1)$ & $1(1)$ & $-1(1)$ & $-2(1)$ \\
\hline $\mathrm{C}(10)$ & $27(1)$ & $33(1)$ & $24(1)$ & $3(1)$ & $0(1)$ & $-6(1)$ \\
\hline $\mathrm{C}(11)$ & $32(1)$ & $27(1)$ & $25(1)$ & $2(1)$ & $-2(1)$ & $-6(1)$ \\
\hline $\mathrm{C}(12)$ & $31(1)$ & $26(1)$ & $24(1)$ & $2(1)$ & $0(1)$ & $0(1)$ \\
\hline $\mathrm{C}(13)$ & $26(1)$ & $26(1)$ & $22(1)$ & 1(1) & $-1(1)$ & $-1(1)$ \\
\hline $\mathrm{C}(14)$ & $27(1)$ & $37(1)$ & $45(1)$ & 1(1) & $0(1)$ & $-6(1)$ \\
\hline$C(15)$ & $41(1)$ & $29(1)$ & $45(1)$ & 1(1) & $1(1)$ & $-9(1)$ \\
\hline$C(16)$ & $44(1)$ & $27(1)$ & $34(1)$ & 1(1) & $3(1)$ & $-1(1)$ \\
\hline $\mathrm{C}(17)$ & $32(1)$ & $30(1)$ & $35(1)$ & $2(1)$ & $-2(1)$ & $5(1)$ \\
\hline $\mathrm{C}(18)$ & $27(1)$ & $37(1)$ & $34(1)$ & $-1(1)$ & $5(1)$ & $0(1)$ \\
\hline $\mathrm{C}(19)$ & $29(1)$ & $44(1)$ & $41(1)$ & $-1(1)$ & $2(1)$ & $-11(1)$ \\
\hline$C(20)$ & $41(1)$ & $30(1)$ & $50(1)$ & 1(1) & $-4(1)$ & $-10(1)$ \\
\hline $\mathrm{C}(21)$ & $37(1)$ & $26(1)$ & $44(1)$ & $0(1)$ & $0(1)$ & $3(1)$ \\
\hline
\end{tabular}


Table 4. Bond lengths [ $\mathrm{\AA}]$ and angles [deg] for $\mathrm{Me}_{8} \mathrm{C}_{13} \mathrm{H}_{2}$.

\begin{tabular}{|c|c|c|c|c|c|}
\hline \multirow[b]{2}{*}{$\mathrm{C}(1)$} & \multicolumn{5}{|c|}{ Bonds } \\
\hline & $\mathrm{C}(13)$ & $1.503(2)$ & $\mathrm{C}(12)$ & $\mathrm{C}(21)$ & $1.511(2)$ \\
\hline $\mathrm{C}(1)$ & $\mathrm{C}(2)$ & $1.505(2)$ & $\mathrm{C}(14)$ & $\mathrm{H}(14 \mathrm{~B})$ & $1.00(2)$ \\
\hline $\mathrm{C}(1)$ & $\mathrm{H}(1 \mathrm{~B})$ & $0.983(16)$ & $\mathrm{C}(14)$ & $\mathrm{H}(14 \mathrm{C})$ & $0.99(2)$ \\
\hline $\mathrm{C}(1)$ & $\mathrm{H}(1 \mathrm{~A})$ & $0.959(15)$ & $\mathrm{C}(14)$ & $\mathrm{H}(14 \mathrm{~A})$ & $0.986(18)$ \\
\hline $\mathrm{C}(2)$ & $\mathrm{C}(3)$ & $1.390(2)$ & $\mathrm{C}(15)$ & $\mathrm{H}(15 \mathrm{C})$ & $0.96(2)$ \\
\hline $\mathrm{C}(2)$ & $\mathrm{C}(7)$ & $1.408(2)$ & $C(15)$ & $\mathrm{H}(15 \mathrm{~B})$ & $0.952(19)$ \\
\hline $\mathrm{C}(3)$ & $\mathrm{C}(4)$ & $1.405(2)$ & $\mathrm{C}(15)$ & $\mathrm{H}(15 \mathrm{~A})$ & $1.02(2)$ \\
\hline $\mathrm{C}(3)$ & $\mathrm{C}(14)$ & $1.507(2)$ & $\mathrm{C}(16)$ & $\mathrm{H}(16 \mathrm{C})$ & $1.01(2)$ \\
\hline $\mathrm{C}(4)$ & $\mathrm{C}(5)$ & $1.404(2)$ & $C(16)$ & $\mathrm{H}(16 \mathrm{~B})$ & $0.989(18)$ \\
\hline$C(4)$ & $\mathrm{C}(15)$ & $1.513(2)$ & $\mathrm{C}(16)$ & $\mathrm{H}(16 \mathrm{~A})$ & $0.961(17)$ \\
\hline $\mathrm{C}(5)$ & $C(6)$ & $1.413(2)$ & $\mathrm{C}(17)$ & $\mathrm{H}(17 \mathrm{C})$ & $1.038(16)$ \\
\hline$C(5)$ & $\mathrm{C}(16)$ & $1.508(2)$ & $\mathrm{C}(17)$ & $\mathrm{H}(17 \mathrm{~B})$ & $0.998(16)$ \\
\hline$C(6)$ & $\mathrm{C}(7)$ & $1.405(2)$ & $\mathrm{C}(17)$ & $\mathrm{H}(17 \mathrm{~A})$ & $1.022(15)$ \\
\hline$C(6)$ & $\mathrm{C}(17)$ & $1.515(2)$ & $\mathrm{C}(18)$ & $\mathrm{H}(18 \mathrm{C})$ & $0.999(15)$ \\
\hline $\mathrm{C}(7)$ & $\mathrm{C}(8)$ & $1.4939(19)$ & $\mathrm{C}(18)$ & $\mathrm{H}(18 \mathrm{~B})$ & $1.026(17)$ \\
\hline $\mathrm{C}(8)$ & $\mathrm{C}(9)$ & $1.4011(19)$ & $\mathrm{C}(18)$ & $\mathrm{H}(18 \mathrm{~A})$ & $1.034(15)$ \\
\hline $\mathrm{C}(8)$ & $\mathrm{C}(13)$ & $1.408(2)$ & $\mathrm{C}(19)$ & $\mathrm{H}(19 \mathrm{C})$ & $0.94(3)$ \\
\hline $\mathrm{C}(9)$ & $\mathrm{C}(10)$ & $1.415(2)$ & $\mathrm{C}(19)$ & $\mathrm{H}(19 \mathrm{~B})$ & $0.91(2)$ \\
\hline $\mathrm{C}(9)$ & $\mathrm{C}(18)$ & $1.514(2)$ & $\mathrm{C}(19)$ & $\mathrm{H}(19 \mathrm{~A})$ & $0.93(2)$ \\
\hline $\mathrm{C}(10)$ & $\mathrm{C}(11)$ & $1.408(2)$ & $\mathrm{C}(20)$ & $\mathrm{H}(20 \mathrm{C})$ & $1.01(2)$ \\
\hline$C(10)$ & C(19) & $1.506(2)$ & $\mathrm{C}(20)$ & $\mathrm{H}(20 \mathrm{~B})$ & $0.970(19)$ \\
\hline $\mathrm{C}(11)$ & $\mathrm{C}(12)$ & $1.412(2)$ & $\mathrm{C}(20)$ & $\mathrm{H}(20 \mathrm{~A})$ & $0.94(3)$ \\
\hline $\mathrm{C}(11)$ & $\mathrm{C}(20)$ & $1.509(2)$ & $\mathrm{C}(21)$ & $\mathrm{H}(21 \mathrm{C})$ & $0.93(3)$ \\
\hline \multirow[t]{2}{*}{$\mathrm{C}(12)$} & $\mathrm{C}(13)$ & $1.3856(19)$ & $\mathrm{C}(21)$ & $\mathrm{H}(21 \mathrm{~B})$ & $0.98(2)$ \\
\hline & & & $\mathrm{C}(21)$ & $H(21 A)$ & $0.95(2)$ \\
\hline
\end{tabular}

$\mathrm{C}(13)-\mathrm{C}(1)-\mathrm{C}(2)$

$\mathrm{C}(13)-\mathrm{C}(1)-\mathrm{H}(1 \mathrm{~B})$

$\mathrm{C}(2)-\mathrm{C}(1)-\mathrm{H}(1 \mathrm{~B})$

$\mathrm{C}(13)-\mathrm{C}(1)-\mathrm{H}(1 \mathrm{~A})$

$\mathrm{C}(2)-\mathrm{C}(1)-\mathrm{H}(1 \mathrm{~A})$

$\mathrm{H}(1 \mathrm{~B})-\mathrm{C}(1)-\mathrm{H}(1 \mathrm{~A})$

$\mathrm{C}(3)-\mathrm{C}(2)-\mathrm{C}(7)$

$\mathrm{C}(3)-\mathrm{C}(2)-\mathrm{C}(1)$

$\mathrm{C}(7)-\mathrm{C}(2)-\mathrm{C}(1)$

$\mathrm{C}(2)-\mathrm{C}(3)-\mathrm{C}(4)$

$\mathrm{C}(2)-\mathrm{C}(3)-\mathrm{C}(14)$

$\mathrm{C}(4)-\mathrm{C}(3)-\mathrm{C}(14)$

$\mathrm{C}(5)-\mathrm{C}(4)-\mathrm{C}(3)$

$\mathrm{C}(5)-\mathrm{C}(4)-\mathrm{C}(15)$

$\mathrm{C}(8)-\mathrm{C}(9)-\mathrm{C}(18)$

$\mathrm{C}(10)-\mathrm{C}(9)-\mathrm{C}(18)$

C(11)-C(10)-C(9)

C(11)-C(10)-C(19)
Angles

$\begin{array}{rlr}103.15(12) & \mathrm{C}(3)-\mathrm{C}(4)-\mathrm{C}(15) & 120.05(14) \\ 110.2(8) & \mathrm{C}(4)-\mathrm{C}(5)-\mathrm{C}(6) & 120.99(13) \\ 113.5(8) & \mathrm{C}(4)-\mathrm{C}(5)-\mathrm{C}(16) & 118.58(14) \\ 111.2(9) & \mathrm{C}(6)-\mathrm{C}(5)-\mathrm{C}(16) & 120.41(14) \\ 111.3(9) & \mathrm{C}(7)-\mathrm{C}(6)-\mathrm{C}(5) & 118.26(13) \\ 107.5(13) & \mathrm{C}(7)-\mathrm{C}(6)-\mathrm{C}(17) & 122.15(13) \\ 122.33(13) & \mathrm{C}(5)-\mathrm{C}(6)-\mathrm{C}(17) & 119.05(13) \\ 127.01(14) & \mathrm{C}(6)-\mathrm{C}(7)-\mathrm{C}(2) & 119.09(13) \\ 110.52(12) & \mathrm{C}(6)-\mathrm{C}(7)-\mathrm{C}(8) & 133.23(13) \\ 118.07(13) & \mathrm{C}(2)-\mathrm{C}(7)-\mathrm{C}(8) & 107.43(12) \\ 119.91(14) & \mathrm{C}(9)-\mathrm{C}(8)-\mathrm{C}(13) & 119.06(13) \\ 121.99(14) & \mathrm{C}(9)-\mathrm{C}(8)-\mathrm{C}(7) & 132.82(13) \\ 120.30(13) & \mathrm{C}(13)-\mathrm{C}(8)-\mathrm{C}(7) & 107.87(12) \\ 119.54(14) & \mathrm{C}(8)-\mathrm{C}(9)-\mathrm{C}(10) & 118.04(13) \\ 122.54(14) & \mathrm{C}(6)-\mathrm{C}(17)-\mathrm{H}(17 \mathrm{~B}) & 113.3(8) \\ 118.91(13) & \mathrm{H}(17 \mathrm{C})-\mathrm{C}(17)-\mathrm{H}(17 \mathrm{~B}) & 108.0(12) \\ 121.24(13) & \mathrm{C}(6)-\mathrm{C}(17)-\mathrm{H}(17 \mathrm{~A}) & 110.2(8) \\ 119.79(14) & \mathrm{H}(17 \mathrm{C})-\mathrm{C}(17)-\mathrm{H}(17 \mathrm{~A}) & 107.6(12)\end{array}$




\begin{tabular}{|c|c|c|c|}
\hline $\mathrm{C}(9)-\mathrm{C}(10)-\mathrm{C}(19)$ & $118.97(14)$ & $\mathrm{H}(17 \mathrm{~B})-\mathrm{C}(17)-\mathrm{H}(17 \mathrm{~A})$ & $108.7(12)$ \\
\hline$C(10)-C(11)-C(12)$ & $120.04(13)$ & $\mathrm{C}(9)-\mathrm{C}(18)-\mathrm{H}(18 \mathrm{C})$ & $110.2(9)$ \\
\hline$C(10)-C(11)-C(20)$ & $120.36(14)$ & $\mathrm{C}(9)-\mathrm{C}(18)-\mathrm{H}(18 \mathrm{~B})$ & $116.0(8)$ \\
\hline$C(12)-C(11)-C(20)$ & $119.52(14)$ & $\mathrm{H}(18 \mathrm{C})-\mathrm{C}(18)-\mathrm{H}(18 \mathrm{~B})$ & $106.0(12)$ \\
\hline$C(13)-C(12)-C(11)$ & $117.59(13)$ & $\mathrm{C}(9)-\mathrm{C}(18)-\mathrm{H}(18 \mathrm{~A})$ & $110.9(8)$ \\
\hline$C(13)-C(12)-C(21)$ & $120.01(14)$ & $\mathrm{H}(18 \mathrm{C})-\mathrm{C}(18)-\mathrm{H}(18 \mathrm{~A})$ & $107.5(11)$ \\
\hline$C(11)-C(12)-C(21)$ & $122.40(14)$ & $\mathrm{H}(18 \mathrm{~B})-\mathrm{C}(18)-\mathrm{H}(18 \mathrm{~A})$ & $105.8(12)$ \\
\hline $\mathrm{C}(12)-\mathrm{C}(13)-\mathrm{C}(8)$ & $122.99(13)$ & $\mathrm{C}(10)-\mathrm{C}(19)-\mathrm{H}(19 \mathrm{C})$ & $113.6(17)$ \\
\hline$C(12)-C(13)-C(1)$ & $126.57(13)$ & $\mathrm{C}(10)-\mathrm{C}(19)-\mathrm{H}(19 \mathrm{~B})$ & $114.6(13)$ \\
\hline $\mathrm{C}(8)-\mathrm{C}(13)-\mathrm{C}(1)$ & $110.31(12)$ & $\mathrm{H}(19 \mathrm{C})-\mathrm{C}(19)-\mathrm{H}(19 \mathrm{~B})$ & $100(2)$ \\
\hline $\mathrm{C}(3)-\mathrm{C}(14)-\mathrm{H}(14 \mathrm{~B})$ & 111.1(11) & $\mathrm{C}(10)-\mathrm{C}(19)-\mathrm{H}(19 \mathrm{~A})$ & $114.5(14)$ \\
\hline $\mathrm{C}(3)-\mathrm{C}(14)-\mathrm{H}(14 \mathrm{C})$ & $111.3(12)$ & $\mathrm{H}(19 \mathrm{C})-\mathrm{C}(19)-\mathrm{H}(19 \mathrm{~A})$ & $105.3(18)$ \\
\hline $\mathrm{H}(14 \mathrm{~B})-\mathrm{C}(14)-\mathrm{H}(14 \mathrm{C})$ & $106.1(17)$ & $\mathrm{H}(19 \mathrm{~B})-\mathrm{C}(19)-\mathrm{H}(19 \mathrm{~A})$ & 107.2(19) \\
\hline $\mathrm{C}(3)-\mathrm{C}(14)-\mathrm{H}(14 \mathrm{~A})$ & $111.9(10)$ & $\mathrm{C}(11)-\mathrm{C}(20)-\mathrm{H}(20 \mathrm{C})$ & $112.9(13)$ \\
\hline $\mathrm{H}(14 \mathrm{~B})-\mathrm{C}(14)-\mathrm{H}(14 \mathrm{~A})$ & $107.3(15)$ & $\mathrm{C}(11)-\mathrm{C}(20)-\mathrm{H}(20 \mathrm{~B})$ & $111.5(11)$ \\
\hline $\mathrm{H}(14 \mathrm{C})-\mathrm{C}(14)-\mathrm{H}(14 \mathrm{~A})$ & $108.8(15)$ & $\mathrm{H}(20 \mathrm{C})-\mathrm{C}(20)-\mathrm{H}(20 \mathrm{~B})$ & $102.5(16)$ \\
\hline $\mathrm{C}(4)-\mathrm{C}(15)-\mathrm{H}(15 \mathrm{C})$ & $109.3(15)$ & $\mathrm{C}(11)-\mathrm{C}(20)-\mathrm{H}(20 \mathrm{~A})$ & $112.6(17)$ \\
\hline $\mathrm{C}(4)-\mathrm{C}(15)-\mathrm{H}(15 \mathrm{~B})$ & $110.6(12)$ & $H(20 C)-C(20)-H(20 A)$ & $108(2)$ \\
\hline $\mathrm{H}(15 \mathrm{C})-\mathrm{C}(15)-\mathrm{H}(15 \mathrm{~B})$ & $109.9(19)$ & $\mathrm{H}(20 \mathrm{~B})-\mathrm{C}(20)-\mathrm{H}(20 \mathrm{~A})$ & $108.9(18)$ \\
\hline $\mathrm{C}(4)-\mathrm{C}(15)-\mathrm{H}(15 \mathrm{~A})$ & $112.7(11)$ & $\mathrm{C}(12)-\mathrm{C}(21)-\mathrm{H}(21 \mathrm{C})$ & $114.8(15)$ \\
\hline $\mathrm{H}(15 \mathrm{C})-\mathrm{C}(15)-\mathrm{H}(15 \mathrm{~A})$ & $110.0(18)$ & $\mathrm{C}(12)-\mathrm{C}(21)-\mathrm{H}(21 \mathrm{~B})$ & $111.5(13)$ \\
\hline $\mathrm{H}(15 \mathrm{~B})-\mathrm{C}(15)-\mathrm{H}(15 \mathrm{~A})$ & $104.2(15)$ & $\mathrm{H}(21 \mathrm{C})-\mathrm{C}(21)-\mathrm{H}(21 \mathrm{~B})$ & $105.5(19)$ \\
\hline $\mathrm{C}(5)-\mathrm{C}(16)-\mathrm{H}(16 \mathrm{C})$ & $112.5(11)$ & $\mathrm{C}(12)-\mathrm{C}(21)-\mathrm{H}(21 \mathrm{~A})$ & $110.8(13)$ \\
\hline $\mathrm{C}(5)-\mathrm{C}(16)-\mathrm{H}(16 \mathrm{~B})$ & $110.8(10)$ & $H(21 C)-C(21)-H(21 A)$ & $108.3(18)$ \\
\hline $\mathrm{H}(16 \mathrm{C})-\mathrm{C}(16)-\mathrm{H}(16 \mathrm{~B})$ & $106.4(14)$ & $H(21 B)-C(21)-H(21 A)$ & $105.5(18)$ \\
\hline $\mathrm{C}(5)-\mathrm{C}(16)-\mathrm{H}(16 \mathrm{~A})$ & $110.8(10)$ & & \\
\hline $\mathrm{H}(16 \mathrm{C})-\mathrm{C}(16)-\mathrm{H}(16 \mathrm{~A})$ & $107.3(14)$ & & \\
\hline $\mathrm{H}(16 \mathrm{~B})-\mathrm{C}(16)-\mathrm{H}(16 \mathrm{~A})$ & $108.8(14)$ & & \\
\hline $\mathrm{C}(6)-\mathrm{C}(17)-\mathrm{H}(17 \mathrm{C})$ & $108.9(8)$ & & \\
\hline
\end{tabular}


Table 5. Torsion angles [deg] for $\mathrm{Me}_{8} \mathrm{C}_{13} \mathrm{H}_{2}$.

\begin{tabular}{|c|c|c|c|}
\hline $\mathrm{C}(13)-\mathrm{C}(1)-\mathrm{C}(2)-\mathrm{C}(3)$ & $178.82(12)$ & $C(2)-C(7)-C(8)-C(13)$ & $8.86(14)$ \\
\hline $\mathrm{C}(13)-\mathrm{C}(1)-\mathrm{C}(2)-\mathrm{C}(7)$ & $3.11(15)$ & $\mathrm{C}(13)-\mathrm{C}(8)-\mathrm{C}(9)-\mathrm{C}(10)$ & $11.59(18)$ \\
\hline $\mathrm{C}(7)-\mathrm{C}(2)-\mathrm{C}(3)-\mathrm{C}(4)$ & $-0.15(19)$ & $\mathrm{C}(7)-\mathrm{C}(8)-\mathrm{C}(9)-\mathrm{C}(10)$ & $-174.97(13)$ \\
\hline $\mathrm{C}(1)-\mathrm{C}(2)-\mathrm{C}(3)-\mathrm{C}(4)$ & $-175.40(13)$ & $\mathrm{C}(13)-\mathrm{C}(8)-\mathrm{C}(9)-\mathrm{C}(18)$ & $-160.12(13)$ \\
\hline$C(7)-C(2)-C(3)-C(14)$ & $177.97(13)$ & $C(7)-C(8)-C(9)-C(18)$ & $13.3(2)$ \\
\hline $\mathrm{C}(1)-\mathrm{C}(2)-\mathrm{C}(3)-\mathrm{C}(14)$ & $2.7(2)$ & $\mathrm{C}(8)-\mathrm{C}(9)-\mathrm{C}(10)-\mathrm{C}(11)$ & $-6.68(19)$ \\
\hline$C(2)-C(3)-C(4)-C(5)$ & $5.39(19)$ & $\mathrm{C}(18)-\mathrm{C}(9)-\mathrm{C}(10)-\mathrm{C}(11)$ & $165.34(13)$ \\
\hline$C(14)-C(3)-C(4)-C(5)$ & $-172.68(14)$ & $\mathrm{C}(8)-\mathrm{C}(9)-\mathrm{C}(10)-\mathrm{C}(19)$ & $173.00(13)$ \\
\hline$C(2)-C(3)-C(4)-C(15)$ & $-178.41(13)$ & $\mathrm{C}(18)-\mathrm{C}(9)-\mathrm{C}(10)-\mathrm{C}(19)$ & $-14.99(19)$ \\
\hline$C(14)-C(3)-C(4)-C(15)$ & $3.5(2)$ & $\mathrm{C}(9)-\mathrm{C}(10)-\mathrm{C}(11)-\mathrm{C}(12)$ & $-2.3(2)$ \\
\hline$C(3)-C(4)-C(5)-C(6)$ & $-2.20(19)$ & $C(19)-C(10)-C(11)-C(12)$ & $178.05(13)$ \\
\hline$C(15)-C(4)-C(5)-C(6)$ & $-178.42(13)$ & $\mathrm{C}(9)-\mathrm{C}(10)-\mathrm{C}(11)-\mathrm{C}(20)$ & $-178.92(13)$ \\
\hline $\mathrm{C}(3)-\mathrm{C}(4)-\mathrm{C}(5)-\mathrm{C}(16)$ & $175.98(12)$ & $\mathrm{C}(19)-\mathrm{C}(10)-\mathrm{C}(11)-\mathrm{C}(20)$ & $1.4(2)$ \\
\hline$C(15)-C(4)-C(5)-C(16)$ & $-0.2(2)$ & $\mathrm{C}(10)-\mathrm{C}(11)-\mathrm{C}(12)-\mathrm{C}(13)$ & $6.03(19)$ \\
\hline$C(4)-C(5)-C(6)-C(7)$ & $-6.24(19)$ & $\mathrm{C}(20)-\mathrm{C}(11)-\mathrm{C}(12)-\mathrm{C}(13)$ & $-177.30(13)$ \\
\hline$C(16)-C(5)-C(6)-C(7)$ & $175.61(12)$ & $C(10)-C(11)-C(12)-C(21)$ & $-173.83(13)$ \\
\hline$C(4)-C(5)-C(6)-C(17)$ & $165.45(13)$ & $\mathrm{C}(20)-\mathrm{C}(11)-\mathrm{C}(12)-\mathrm{C}(21)$ & $2.8(2)$ \\
\hline$C(16)-C(5)-C(6)-C(17)$ & $-12.70(19)$ & $\mathrm{C}(11)-\mathrm{C}(12)-\mathrm{C}(13)-\mathrm{C}(8)$ & $-0.92(19)$ \\
\hline$C(5)-C(6)-C(7)-C(2)$ & $11.26(18)$ & $\mathrm{C}(21)-\mathrm{C}(12)-\mathrm{C}(13)-\mathrm{C}(8)$ & $178.95(13)$ \\
\hline$C(17)-C(6)-C(7)-C(2)$ & $-160.15(13)$ & $\mathrm{C}(11)-\mathrm{C}(12)-\mathrm{C}(13)-\mathrm{C}(1)$ & $-176.27(13)$ \\
\hline$C(5)-C(6)-C(7)-C(8)$ & $-175.37(13)$ & $\mathrm{C}(21)-\mathrm{C}(12)-\mathrm{C}(13)-\mathrm{C}(1)$ & $3.6(2)$ \\
\hline $\mathrm{C}(17)-\mathrm{C}(6)-\mathrm{C}(7)-\mathrm{C}(8)$ & $13.2(2)$ & $\mathrm{C}(9)-\mathrm{C}(8)-\mathrm{C}(13)-\mathrm{C}(12)$ & $-8.08(19)$ \\
\hline$C(3)-C(2)-C(7)-C(6)$ & $-8.32(19)$ & $C(7)-C(8)-C(13)-C(12)$ & $176.97(12)$ \\
\hline$C(1)-C(2)-C(7)-C(6)$ & $167.63(12)$ & $\mathrm{C}(9)-\mathrm{C}(8)-\mathrm{C}(13)-\mathrm{C}(1)$ & $167.95(12)$ \\
\hline$C(3)-C(2)-C(7)-C(8)$ & $176.74(12)$ & $C(7)-C(8)-C(13)-C(1)$ & $-7.01(14)$ \\
\hline $\mathrm{C}(1)-\mathrm{C}(2)-\mathrm{C}(7)-\mathrm{C}(8)$ & $-7.31(14)$ & $\mathrm{C}(2)-\mathrm{C}(1)-\mathrm{C}(13)-\mathrm{C}(12)$ & $178.45(12)$ \\
\hline$C(6)-C(7)-C(8)-C(9)$ & $21.0(2)$ & $\mathrm{C}(2)-\mathrm{C}(1)-\mathrm{C}(13)-\mathrm{C}(8)$ & $2.60(14)$ \\
\hline$C(2)-C(7)-C(8)-C(9)$ & $-165.12(14)$ & & \\
\hline $\mathrm{C}(6)-\mathrm{C}(7)-\mathrm{C}(8)-\mathrm{C}(13)$ & $-165.06(14)$ & & \\
\hline
\end{tabular}




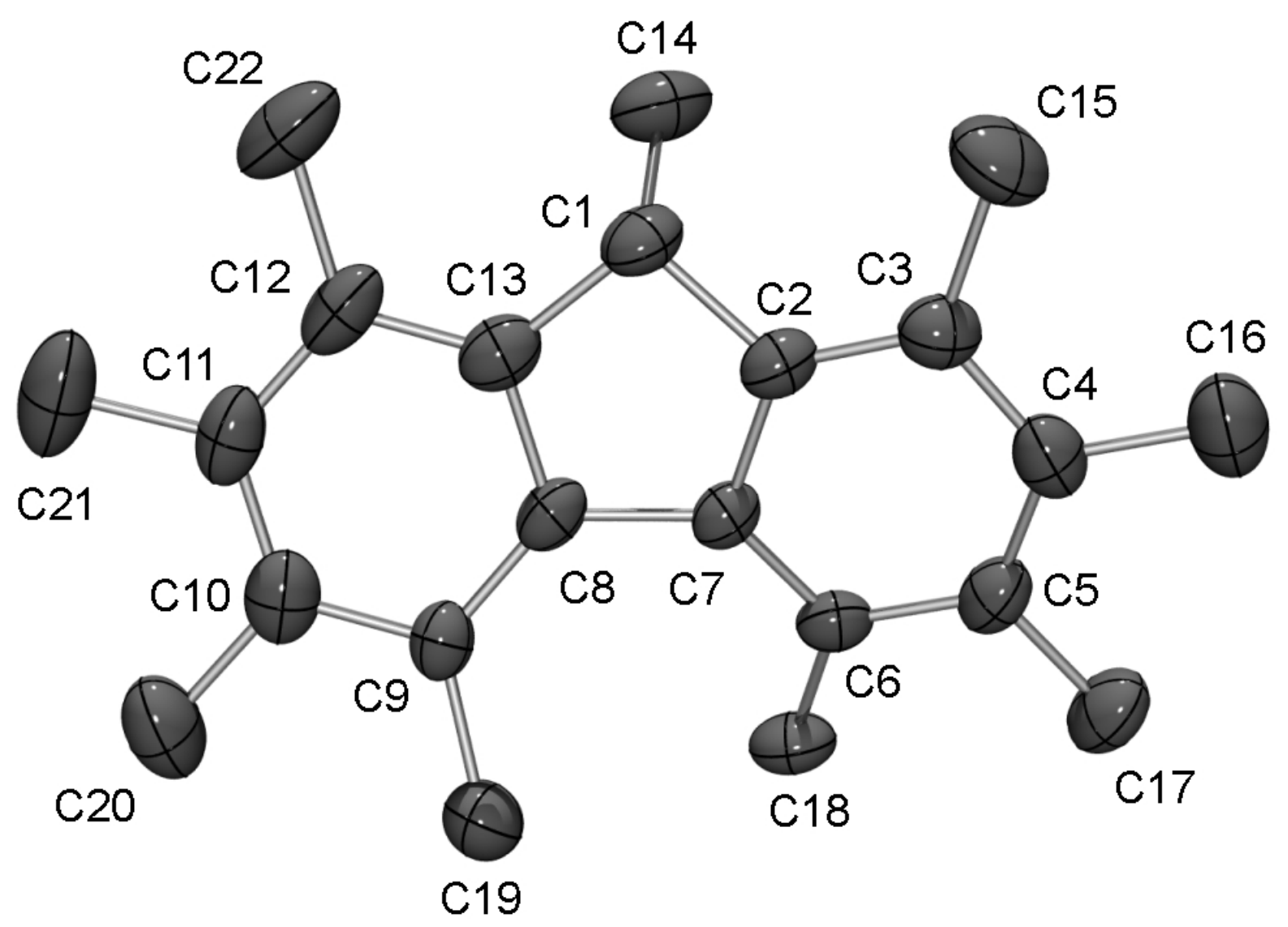

Figure 2. Thermal ellipsoid plot for compound $\mathrm{C}_{13} \mathrm{Me}_{9} \mathrm{H}(2)$. Hydrogen atoms are omitted for clarity. Thermal ellipsoids are drawn at $50 \%$ probability. 


\section{EXPERIMENTAL DETAILS}

\section{Crystal Data}

Empirical Formula

Formula Weight

Crystal Color, Habit

Crystal Dimensions

Crystal System

Lattice Type

Lattice Parameters
$\mathrm{C}_{22} \mathrm{H}_{28}$

292.44

colorless, block

$0.25 \times 0.11 \times 0.06 \mathrm{~mm}$

monoclinic

C-centered

$a=23.89(2) \AA$

$b=9.191(9) \AA$

$c=15.890(14) \AA$

$\beta=101.06(2)^{\mathrm{O}}$

$V=3424(5) \AA^{3}$

C2/c (\#15)

8

$1.135 \mathrm{~g} / \mathrm{cm}^{3}$

1280.00

$0.063 \mathrm{~mm}^{-1}$ 


\section{E. Intensity Measurements}

Diffractometer

Radiation

Detector Position

Exposure Time

Scan Type

$2 \theta_{\max }$

No. of Reflections Measured

Corrections
Bruker APEX CCD

$\operatorname{MoK} \alpha(\lambda=0.71073 \AA)$

graphite monochromated

$60.00 \mathrm{~mm}$

15.0 seconds per frame.

$\omega(0.3$ degrees per frame $)$

$46.5^{\circ}$

Total: 7453

Unique: $2461\left(\mathrm{R}_{\mathrm{int}}=0.075\right)$

Lorentz-polarization

Absorption $\left(\mathrm{T}_{\max }=1.00 \mathrm{~T}_{\min }=0.91\right)$ 


\section{F. Structure Solution and Refinement}

Structure Solution

Refinement

Function Minimized

Least Squares Weights

p-factor

Anomalous Dispersion

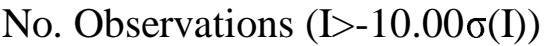

No. Variables

Reflection/Parameter Ratio

Residuals: R; Rw; Rall

Goodness of Fit Indicator

Max Shift/Error in Final Cycle

Maximum peak in Final Diff. Map

Minimum peak in Final Diff. Map
Direct Methods (SIR92)

Full-matrix least-squares

$\Sigma \mathrm{w}(|\mathrm{Fo}|-|\mathrm{Fc}|)^{2}$

$1 / \sigma^{2}(\mathrm{Fo})=4 \mathrm{Fo}^{2} / \sigma^{2}\left(\mathrm{Fo}^{2}\right)$

0.12

All non-hydrogen atoms

2461

199

12.37

$0.0762 ; 0.2175 ; 0.1228$

1.219

0.00

$0.34 \mathrm{e}^{-/} \AA^{3}$

$-0.26 \mathrm{e}^{-} / \AA^{3}$ 


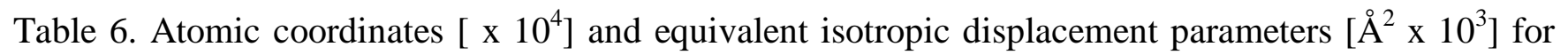
$\mathbf{C}_{\mathbf{1 3}} \mathbf{M e}_{9} \mathbf{H}$. U(eq) is defined as one third of the trace of the orthogonalized $\mathrm{U}_{\mathrm{ij}}$ tensor.

\begin{tabular}{lcccc}
\hline & & & & \\
& $\mathrm{X}$ & $\mathrm{y}$ & $\mathrm{U}(\mathrm{eq})$ \\
\hline $\mathrm{C}(1)$ & $1319(2)$ & $2732(4)$ & $-3253(2)$ & $43(1)$ \\
$\mathrm{C}(2)$ & $1516(2)$ & $1423(4)$ & $-2691(3)$ & $43(1)$ \\
$\mathrm{C}(3)$ & $1769(2)$ & $1425(4)$ & $-1838(3)$ & $48(1)$ \\
$\mathrm{C}(4)$ & $1950(2)$ & $81(5)$ & $-1442(3)$ & $49(1)$ \\
$\mathrm{C}(5)$ & $1895(2)$ & $-1183(5)$ & $-1935(3)$ & $48(1)$ \\
$\mathrm{C}(6)$ & $1652(2)$ & $-1175(4)$ & $-2819(2)$ & $37(1)$ \\
$\mathrm{C}(7)$ & $1426(2)$ & $138(4)$ & $-3182(2)$ & $35(1)$ \\
$\mathrm{C}(8)$ & $1143(2)$ & $543(4)$ & $-4074(2)$ & $33(1)$ \\
$\mathrm{C}(9)$ & $855(2)$ & $-255(4)$ & $-4778(2)$ & $36(1)$ \\
$\mathrm{C}(10)$ & $693(2)$ & $467(4)$ & $-5576(2)$ & $42(1)$ \\
$\mathrm{C}(11)$ & $782(2)$ & $1966(4)$ & $-5648(3)$ & $44(1)$ \\
$\mathrm{C}(12)$ & $994(2)$ & $2785(4)$ & $-4907(3)$ & $43(1)$ \\
$\mathrm{C}(13)$ & $1159(2)$ & $2066(4)$ & $-4135(2)$ & $38(1)$ \\
$\mathrm{C}(14)$ & $797(2)$ & $3487(4)$ & $-3007(3)$ & $61(1)$ \\
$\mathrm{C}(15)$ & $1863(2)$ & $2826(5)$ & $-1339(3)$ & $69(2)$ \\
$\mathrm{C}(16)$ & $2215(2)$ & $24(6)$ & $-502(3)$ & $74(2)$ \\
$\mathrm{C}(17)$ & $2106(2)$ & $-2610(5)$ & $-1510(3)$ & $65(1)$ \\
$\mathrm{C}(18)$ & $1700(2)$ & $-2531(4)$ & $-3329(3)$ & $52(1)$ \\
$\mathrm{C}(19)$ & $646(2)$ & $-1790(4)$ & $-4707(3)$ & $47(1)$ \\
$\mathrm{C}(20)$ & $419(2)$ & $-374(5)$ & $-6353(3)$ & $55(1)$ \\
$\mathrm{C}(21)$ & $586(2)$ & $2715(5)$ & $-6495(3)$ & $62(1)$ \\
$\mathrm{C}(22)$ & $1032(2)$ & $4425(4)$ & $-4957(3)$ & $65(1)$ \\
& & & & \\
\hline
\end{tabular}


Table 7. Hydrogen coordinates [ $\mathrm{x} 10^{4]}$ and isotropic displacement parameters $\left[\AA^{2} \times 10^{3}\right]$ for $\mathbf{C}_{\mathbf{1 3}} \mathbf{M} \mathbf{e}_{9} \mathbf{H}$.

\begin{tabular}{|c|c|c|c|c|}
\hline & $\mathrm{x}$ & $\mathrm{y}$ & $\mathrm{z}$ & $\mathrm{U}(\mathrm{eq})$ \\
\hline$\overline{H(1 A)}$ & 1633 & 3427 & -3234 & 52 \\
\hline $\mathrm{H}(14 \mathrm{~A})$ & 674 & 4270 & -3399 & 91 \\
\hline $\mathrm{H}(14 \mathrm{~B})$ & 898 & 3866 & -2435 & 91 \\
\hline $\mathrm{H}(14 \mathrm{C})$ & 492 & 2796 & -3034 & 91 \\
\hline $\mathrm{H}(15 \mathrm{~A})$ & 2050 & 2624 & -761 & 103 \\
\hline $\mathrm{H}(15 \mathrm{~B})$ & 1503 & 3284 & -1337 & 103 \\
\hline $\mathrm{H}(15 \mathrm{C})$ & 2098 & 3463 & -1603 & 103 \\
\hline $\mathrm{H}(16 \mathrm{~A})$ & 2218 & 982 & -261 & 111 \\
\hline $\mathrm{H}(16 \mathrm{~B})$ & 2599 & -331 & -435 & 111 \\
\hline $\mathrm{H}(16 \mathrm{C})$ & 1997 & -615 & -211 & 111 \\
\hline $\mathrm{H}(17 \mathrm{~A})$ & 2253 & -2447 & -912 & 98 \\
\hline $\mathrm{H}(17 \mathrm{~B})$ & 2403 & -2995 & -1776 & 98 \\
\hline $\mathrm{H}(17 \mathrm{C})$ & 1796 & -3291 & -1573 & 98 \\
\hline $\mathrm{H}(18 \mathrm{~A})$ & 1865 & -3296 & -2950 & 78 \\
\hline $\mathrm{H}(18 \mathrm{~B})$ & 1938 & -2342 & -3740 & 78 \\
\hline $\mathrm{H}(18 \mathrm{C})$ & 1327 & -2821 & -3623 & 78 \\
\hline $\mathrm{H}(19 \mathrm{~A})$ & 793 & -2159 & -4143 & 71 \\
\hline $\mathrm{H}(19 \mathrm{~B})$ & 774 & -2392 & -5126 & 71 \\
\hline $\mathrm{H}(19 \mathrm{C})$ & 236 & -1794 & -4808 & 71 \\
\hline $\mathrm{H}(20 \mathrm{~A})$ & 389 & -1379 & -6203 & 82 \\
\hline $\mathrm{H}(20 \mathrm{~B})$ & 647 & -292 & -6787 & 82 \\
\hline $\mathrm{H}(20 \mathrm{C})$ & 45 & 9 & -6567 & 82 \\
\hline $\mathrm{H}(21 \mathrm{~A})$ & 696 & 3720 & -6445 & 93 \\
\hline $\mathrm{H}(21 \mathrm{~B})$ & 179 & 2646 & -6656 & 93 \\
\hline $\mathrm{H}(21 \mathrm{C})$ & 759 & 2256 & -6924 & 93 \\
\hline $\mathrm{H}(22 \mathrm{~A})$ & 1182 & 4809 & -4397 & 97 \\
\hline $\mathrm{H}(22 \mathrm{~B})$ & 659 & 4821 & -5163 & 97 \\
\hline $\mathrm{H}(22 \mathrm{C})$ & 1280 & 4686 & -5342 & 97 \\
\hline
\end{tabular}


Table 8. Anisotropic displacement parameters $\left[\AA^{2} \times 10^{3}\right]$ for $\mathbf{C}_{13} \mathbf{M e} \mathbf{9} \mathbf{H}$. The anisotropic displacement factor exponent takes the form: $-2 \pi^{2}\left[(h a *)^{2} U_{11}+\ldots+2 h k a * b * U_{12}\right]$

\begin{tabular}{lcccccl}
\hline & & & & \\
& $\mathrm{U}_{11}$ & $\mathrm{U}_{22}$ & $\mathrm{U}_{33}$ & $\mathrm{U}_{23}$ & $\mathrm{U}_{13}$ & $\mathrm{U}_{12}$ \\
\hline $\mathrm{C}(1)$ & $52(3)$ & $35(2)$ & $44(3)$ & $-11(2)$ & $10(2)$ & $-10(2)$ \\
$\mathrm{C}(2)$ & $42(3)$ & $44(2)$ & $45(3)$ & $-9(2)$ & $14(2)$ & $-8(2)$ \\
$\mathrm{C}(3)$ & $40(3)$ & $67(3)$ & $38(3)$ & $-19(2)$ & $11(2)$ & $-11(2)$ \\
$\mathrm{C}(4)$ & $37(3)$ & $74(3)$ & $34(3)$ & $-2(2)$ & $7(2)$ & $-4(2)$ \\
$\mathrm{C}(5)$ & $37(3)$ & $65(3)$ & $43(3)$ & $3(2)$ & $11(2)$ & $-1(2)$ \\
$\mathrm{C}(6)$ & $35(2)$ & $43(2)$ & $32(2)$ & $1(2)$ & $7(2)$ & $-3(2)$ \\
$\mathrm{C}(7)$ & $34(2)$ & $42(2)$ & $32(2)$ & $-8(2)$ & $11(2)$ & $-7(2)$ \\
$\mathrm{C}(8)$ & $32(2)$ & $35(2)$ & $32(2)$ & $-5(2)$ & $10(2)$ & $-5(2)$ \\
$\mathrm{C}(9)$ & $36(2)$ & $32(2)$ & $42(3)$ & $-7(2)$ & $15(2)$ & $-1(2)$ \\
$\mathrm{C}(10)$ & $36(2)$ & $51(3)$ & $37(3)$ & $-9(2)$ & $5(2)$ & $-1(2)$ \\
$\mathrm{C}(11)$ & $41(3)$ & $47(2)$ & $46(3)$ & $9(2)$ & $13(2)$ & $6(2)$ \\
$\mathrm{C}(12)$ & $47(3)$ & $36(2)$ & $49(3)$ & $-2(2)$ & $15(2)$ & $-2(2)$ \\
$\mathrm{C}(13)$ & $40(3)$ & $36(2)$ & $39(3)$ & $-8(2)$ & $12(2)$ & $-6(2)$ \\
$\mathrm{C}(14)$ & $76(4)$ & $44(2)$ & $67(3)$ & $-17(2)$ & $30(3)$ & $-5(2)$ \\
$\mathrm{C}(15)$ & $60(3)$ & $86(4)$ & $60(3)$ & $-38(3)$ & $12(3)$ & $-13(3)$ \\
$\mathrm{C}(16)$ & $51(3)$ & $125(5)$ & $43(3)$ & $-4(3)$ & $2(3)$ & $-1(3)$ \\
$\mathrm{C}(17)$ & $61(3)$ & $75(3)$ & $61(3)$ & $20(3)$ & $15(3)$ & $15(3)$ \\
$\mathrm{C}(18)$ & $58(3)$ & $45(2)$ & $53(3)$ & $1(2)$ & $11(2)$ & $6(2)$ \\
$\mathrm{C}(19)$ & $48(3)$ & $36(2)$ & $58(3)$ & $-15(2)$ & $13(2)$ & $-3(2)$ \\
$\mathrm{C}(20)$ & $49(3)$ & $66(3)$ & $45(3)$ & $-16(2)$ & $1(2)$ & $-1(2)$ \\
$\mathrm{C}(21)$ & $63(3)$ & $70(3)$ & $54(3)$ & $14(2)$ & $12(3)$ & $10(3)$ \\
$\mathrm{C}(22)$ & $79(4)$ & $39(2)$ & $77(3)$ & $11(2)$ & $18(3)$ & $-1(2)$ \\
& & & & & & \\
\hline
\end{tabular}


Table 9. Bond lengths $[\AA]$ and angles [deg] for $\mathbf{C}_{\mathbf{1 3}} \mathbf{M} \mathbf{M e}_{9} \mathbf{H}$.

\section{Bonds}

$\mathrm{C}(1)-\mathrm{C}(13) \quad 1.511(5)$

$\mathrm{C}(1)-\mathrm{C}(2) \quad 1.519(6)$

$\mathrm{C}(1)-\mathrm{C}(14) \quad 1.543(6)$

$\mathrm{C}(1)-\mathrm{H}(1 \mathrm{~A}) \quad 0.9800$

$\mathrm{C}(2)-\mathrm{C}(3) \quad 1.374(6)$

$\mathrm{C}(2)-\mathrm{C}(7) \quad 1.409(5)$

$\mathrm{C}(3)-\mathrm{C}(4) \quad 1.416(6)$

$\mathrm{C}(3)-\mathrm{C}(15) \quad 1.506(6)$

$\mathrm{C}(4)-\mathrm{C}(5) \quad 1.393(6)$

$\mathrm{C}(4)-\mathrm{C}(16) \quad 1.507(6)$

$\mathrm{C}(5)-\mathrm{C}(6) \quad 1.414(6)$

$\mathrm{C}(5)-\mathrm{C}(17) \quad 1.516(6)$

$\mathrm{C}(6)-\mathrm{C}(7) \quad 1.401(5)$

$\mathrm{C}(6)-\mathrm{C}(18) \quad 1.503(5)$

$\mathrm{C}(7)-\mathrm{C}(8) \quad 1.495(5)$

$\mathrm{C}(8)-\mathrm{C}(9) \quad 1.404(5)$

$\mathrm{C}(8)-\mathrm{C}(13) \quad 1.404(5)$

$\mathrm{C}(9)-\mathrm{C}(10) \quad 1.417(5)$

$\mathrm{C}(9)-\mathrm{C}(19) \quad 1.508(5)$

$\mathrm{C}(10)-\mathrm{C}(11) \quad 1.402(6)$

$\mathrm{C}(10)-\mathrm{C}(20) \quad 1.497(5)$

$\mathrm{C}(11)-\mathrm{C}(12) \quad 1.407(6)$

$\mathrm{C}(11)-\mathrm{C}(21) \quad 1.504(6)$

$\mathrm{C}(12)-\mathrm{C}(13) \quad 1.382(5)$

$\mathrm{C}(12)-\mathrm{C}(22) \quad 1.513(6)$

$\mathrm{C}(14)-\mathrm{H}(14 \mathrm{~A}) \quad 0.9600$

$\mathrm{C}(14)-\mathrm{H}(14 \mathrm{~B}) \quad 0.9600$

$\mathrm{C}(14)-\mathrm{H}(14 \mathrm{C}) \quad 0.9600$

$\mathrm{C}(15)-\mathrm{H}(15 \mathrm{~A}) \quad 0.9600$

$\mathrm{C}(15)-\mathrm{H}(15 \mathrm{~B}) \quad 0.9600$

$\mathrm{C}(15)-\mathrm{H}(15 \mathrm{C}) \quad 0.9600$

$\mathrm{C}(16)-\mathrm{H}(16 \mathrm{~A}) \quad 0.9600$

$\mathrm{C}(16)-\mathrm{H}(16 \mathrm{~B}) \quad 0.9600$

$\mathrm{C}(16)-\mathrm{H}(16 \mathrm{C}) \quad 0.9600$

$\mathrm{C}(17)-\mathrm{H}(17 \mathrm{~A}) \quad 0.9600$

$\mathrm{C}(17)-\mathrm{H}(17 \mathrm{~B}) \quad 0.9600$

$\mathrm{C}(17)-\mathrm{H}(17 \mathrm{C}) \quad 0.9600$

$\mathrm{C}(18)-\mathrm{H}(18 \mathrm{~A}) \quad 0.9600$

$\mathrm{C}(18)-\mathrm{H}(18 \mathrm{~B}) \quad 0.9600$

$\mathrm{C}(18)-\mathrm{H}(18 \mathrm{C}) \quad 0.9600$

C(19)-H(19A) $\quad 0.9600$

$\mathrm{C}(19)-\mathrm{H}(19 \mathrm{~B}) \quad 0.9600$

$\mathrm{C}(19)-\mathrm{H}(19 \mathrm{C}) \quad 0.9600$

$\mathrm{C}(20)-\mathrm{H}(20 \mathrm{~A}) \quad 0.9600$

$\mathrm{C}(20)-\mathrm{H}(20 \mathrm{~B}) \quad 0.9600$ 


\begin{tabular}{|c|c|}
\hline & \\
\hline $\mathrm{C}(20)-\mathrm{H}(20 \mathrm{C})$ & 0.9600 \\
\hline $\mathrm{C}(21)-\mathrm{H}(21 \mathrm{~A})$ & 0.9600 \\
\hline $\mathrm{C}(21)-\mathrm{H}(21 \mathrm{~B})$ & 0.9600 \\
\hline $\mathrm{C}(21)-\mathrm{H}(21 \mathrm{C})$ & 0.9600 \\
\hline $\mathrm{C}(22)-\mathrm{H}(22 \mathrm{~A})$ & 0.9600 \\
\hline $\mathrm{C}(22)-\mathrm{H}(22 \mathrm{~B})$ & 0.9600 \\
\hline $\mathrm{C}(22)-\mathrm{H}(22 \mathrm{C})$ & 0.9600 \\
\hline \multicolumn{2}{|c|}{ Angles } \\
\hline$C(13)-C(1)-C(2)$ & $102.7(3)$ \\
\hline$C(13)-C(1)-C(14)$ & $110.1(3)$ \\
\hline$C(2)-C(1)-C(14)$ & $112.5(3)$ \\
\hline $\mathrm{C}(13)-\mathrm{C}(1)-\mathrm{H}(1 \mathrm{~A})$ & 110.5 \\
\hline $\mathrm{C}(2)-\mathrm{C}(1)-\mathrm{H}(1 \mathrm{~A})$ & 110.5 \\
\hline $\mathrm{C}(14)-\mathrm{C}(1)-\mathrm{H}(1 \mathrm{~A})$ & 110.5 \\
\hline$C(3)-C(2)-C(7)$ & $122.6(4)$ \\
\hline$C(3)-C(2)-C(1)$ & $127.4(3)$ \\
\hline$C(7)-C(2)-C(1)$ & $110.0(4)$ \\
\hline$C(2)-C(3)-C(4)$ & $118.5(4)$ \\
\hline $\mathrm{C}(2)-\mathrm{C}(3)-\mathrm{C}(15)$ & $120.8(4)$ \\
\hline$C(4)-C(3)-C(15)$ & $120.7(4)$ \\
\hline $\mathrm{C}(5)-\mathrm{C}(4)-\mathrm{C}(3)$ & $119.3(4)$ \\
\hline$C(5)-C(4)-C(16)$ & $120.3(4)$ \\
\hline$C(3)-C(4)-C(16)$ & $120.3(4)$ \\
\hline$C(4)-C(5)-C(6)$ & $122.1(4)$ \\
\hline$C(4)-C(5)-C(17)$ & $118.9(4)$ \\
\hline $\mathrm{C}(6)-\mathrm{C}(5)-\mathrm{C}(17)$ & $119.0(4)$ \\
\hline $\mathrm{C}(7)-\mathrm{C}(6)-\mathrm{C}(5)$ & $117.8(3)$ \\
\hline $\mathrm{C}(7)-\mathrm{C}(6)-\mathrm{C}(18)$ & $123.8(3)$ \\
\hline$C(5)-C(6)-C(18)$ & $118.2(4)$ \\
\hline $\mathrm{C}(6)-\mathrm{C}(7)-\mathrm{C}(2)$ & $119.2(4)$ \\
\hline$C(6)-C(7)-C(8)$ & $132.3(3)$ \\
\hline$C(2)-C(7)-C(8)$ & $108.0(3)$ \\
\hline $\mathrm{C}(9)-\mathrm{C}(8)-\mathrm{C}(13)$ & $118.7(3)$ \\
\hline $\mathrm{C}(9)-\mathrm{C}(8)-\mathrm{C}(7)$ & $133.6(3)$ \\
\hline$C(13)-C(8)-C(7)$ & $107.5(3)$ \\
\hline $\mathrm{C}(8)-\mathrm{C}(9)-\mathrm{C}(10)$ & $118.5(3)$ \\
\hline $\mathrm{C}(8)-\mathrm{C}(9)-\mathrm{C}(19)$ & $123.2(3)$ \\
\hline$C(10)-C(9)-C(19)$ & $117.9(3)$ \\
\hline$C(11)-C(10)-C(9)$ & $120.9(3)$ \\
\hline$C(11)-C(10)-C(20)$ & $119.3(4)$ \\
\hline$C(9)-C(10)-C(20)$ & $119.8(4)$ \\
\hline $\mathrm{C}(10)-\mathrm{C}(11)-\mathrm{C}(12)$ & $119.5(3)$ \\
\hline$C(10)-C(11)-C(21)$ & $119.8(4)$ \\
\hline$C(12)-C(11)-C(21)$ & $120.3(4)$ \\
\hline $\mathrm{C}(13)-\mathrm{C}(12)-\mathrm{C}(11)$ & $118.8(4)$ \\
\hline$C(13)-C(12)-C(22)$ & $120.8(4)$ \\
\hline $\mathrm{C}(11)-\mathrm{C}(12)-\mathrm{C}(22)$ & $120.3(4)$ \\
\hline$C(12)-C(13)-C(8)$ & $122.1(3)$ \\
\hline$C(12)-C(13)-C(1)$ & $127.4(4)$ \\
\hline
\end{tabular}




\begin{tabular}{|c|c|}
\hline $\mathrm{C}(8)-\mathrm{C}(13)-\mathrm{C}(1)$ & $110.2(3)$ \\
\hline $\mathrm{C}(1)-\mathrm{C}(14)-\mathrm{H}(14 \mathrm{~A})$ & 109.5 \\
\hline $\mathrm{C}(1)-\mathrm{C}(14)-\mathrm{H}(14 \mathrm{~B})$ & 109.5 \\
\hline $\mathrm{H}(14 \mathrm{~A})-\mathrm{C}(14)-\mathrm{H}(14 \mathrm{~B})$ & 109.5 \\
\hline $\mathrm{C}(1)-\mathrm{C}(14)-\mathrm{H}(14 \mathrm{C})$ & 109.5 \\
\hline $\mathrm{H}(14 \mathrm{~A})-\mathrm{C}(14)-\mathrm{H}(14 \mathrm{C})$ & 109.5 \\
\hline $\mathrm{H}(14 \mathrm{~B})-\mathrm{C}(14)-\mathrm{H}(14 \mathrm{C})$ & 109.5 \\
\hline $\mathrm{C}(3)-\mathrm{C}(15)-\mathrm{H}(15 \mathrm{~A})$ & 109.5 \\
\hline $\mathrm{C}(3)-\mathrm{C}(15)-\mathrm{H}(15 \mathrm{~B})$ & 109.5 \\
\hline $\mathrm{H}(15 \mathrm{~A})-\mathrm{C}(15)-\mathrm{H}(15 \mathrm{~B})$ & 109.5 \\
\hline $\mathrm{C}(3)-\mathrm{C}(15)-\mathrm{H}(15 \mathrm{C})$ & 109.5 \\
\hline $\mathrm{H}(15 \mathrm{~A})-\mathrm{C}(15)-\mathrm{H}(15 \mathrm{C})$ & 109.5 \\
\hline $\mathrm{H}(15 \mathrm{~B})-\mathrm{C}(15)-\mathrm{H}(15 \mathrm{C})$ & 109.5 \\
\hline $\mathrm{C}(4)-\mathrm{C}(16)-\mathrm{H}(16 \mathrm{~A})$ & 109.5 \\
\hline $\mathrm{C}(4)-\mathrm{C}(16)-\mathrm{H}(16 \mathrm{~B})$ & 109.5 \\
\hline $\mathrm{H}(16 \mathrm{~A})-\mathrm{C}(16)-\mathrm{H}(16 \mathrm{~B})$ & 109.5 \\
\hline $\mathrm{C}(4)-\mathrm{C}(16)-\mathrm{H}(16 \mathrm{C})$ & 109.5 \\
\hline $\mathrm{H}(16 \mathrm{~A})-\mathrm{C}(16)-\mathrm{H}(16 \mathrm{C})$ & 109.5 \\
\hline $\mathrm{H}(16 \mathrm{~B})-\mathrm{C}(16)-\mathrm{H}(16 \mathrm{C})$ & 109.5 \\
\hline $\mathrm{C}(5)-\mathrm{C}(17)-\mathrm{H}(17 \mathrm{~A})$ & 109.5 \\
\hline $\mathrm{C}(5)-\mathrm{C}(17)-\mathrm{H}(17 \mathrm{~B})$ & 109.5 \\
\hline $\mathrm{H}(17 \mathrm{~A})-\mathrm{C}(17)-\mathrm{H}(17 \mathrm{~B})$ & 109.5 \\
\hline $\mathrm{C}(5)-\mathrm{C}(17)-\mathrm{H}(17 \mathrm{C})$ & 109.5 \\
\hline $\mathrm{H}(17 \mathrm{~A})-\mathrm{C}(17)-\mathrm{H}(17 \mathrm{C})$ & 109.5 \\
\hline $\mathrm{H}(17 \mathrm{~B})-\mathrm{C}(17)-\mathrm{H}(17 \mathrm{C})$ & 109.5 \\
\hline $\mathrm{C}(6)-\mathrm{C}(18)-\mathrm{H}(18 \mathrm{~A})$ & 109.5 \\
\hline $\mathrm{C}(6)-\mathrm{C}(18)-\mathrm{H}(18 \mathrm{~B})$ & 109.5 \\
\hline $\mathrm{H}(18 \mathrm{~A})-\mathrm{C}(18)-\mathrm{H}(18 \mathrm{~B})$ & 109.5 \\
\hline $\mathrm{C}(6)-\mathrm{C}(18)-\mathrm{H}(18 \mathrm{C})$ & 109.5 \\
\hline $\mathrm{H}(18 \mathrm{~A})-\mathrm{C}(18)-\mathrm{H}(18 \mathrm{C})$ & 109.5 \\
\hline $\mathrm{H}(18 \mathrm{~B})-\mathrm{C}(18)-\mathrm{H}(18 \mathrm{C})$ & 109.5 \\
\hline $\mathrm{C}(9)-\mathrm{C}(19)-\mathrm{H}(19 \mathrm{~A})$ & 109.5 \\
\hline $\mathrm{C}(9)-\mathrm{C}(19)-\mathrm{H}(19 \mathrm{~B})$ & 109.5 \\
\hline $\mathrm{H}(19 \mathrm{~A})-\mathrm{C}(19)-\mathrm{H}(19 \mathrm{~B})$ & 109.5 \\
\hline $\mathrm{C}(9)-\mathrm{C}(19)-\mathrm{H}(19 \mathrm{C})$ & 109.5 \\
\hline $\mathrm{H}(19 \mathrm{~A})-\mathrm{C}(19)-\mathrm{H}(19 \mathrm{C})$ & 109.5 \\
\hline $\mathrm{H}(19 \mathrm{~B})-\mathrm{C}(19)-\mathrm{H}(19 \mathrm{C})$ & 109.5 \\
\hline $\mathrm{C}(10)-\mathrm{C}(20)-\mathrm{H}(20 \mathrm{~A})$ & 109.5 \\
\hline $\mathrm{C}(10)-\mathrm{C}(20)-\mathrm{H}(20 \mathrm{~B})$ & 109.5 \\
\hline $\mathrm{H}(20 \mathrm{~A})-\mathrm{C}(20)-\mathrm{H}(20 \mathrm{~B})$ & 109.5 \\
\hline $\mathrm{C}(10)-\mathrm{C}(20)-\mathrm{H}(20 \mathrm{C})$ & 109.5 \\
\hline $\mathrm{H}(20 \mathrm{~A})-\mathrm{C}(20)-\mathrm{H}(20 \mathrm{C})$ & 109.5 \\
\hline $\mathrm{H}(20 \mathrm{~B})-\mathrm{C}(20)-\mathrm{H}(20 \mathrm{C})$ & 109.5 \\
\hline $\mathrm{C}(11)-\mathrm{C}(21)-\mathrm{H}(21 \mathrm{~A})$ & 109.5 \\
\hline $\mathrm{C}(11)-\mathrm{C}(21)-\mathrm{H}(21 \mathrm{~B})$ & 109.5 \\
\hline$H(21 A)-C(21)-H(21 B)$ & 109.5 \\
\hline $\mathrm{C}(11)-\mathrm{C}(21)-\mathrm{H}(21 \mathrm{C})$ & 109.5 \\
\hline $\mathrm{H}(21 \mathrm{~A})-\mathrm{C}(21)-\mathrm{H}(21 \mathrm{C})$ & 109.5 \\
\hline $\mathrm{H}(21 \mathrm{~B})-\mathrm{C}(21)-\mathrm{H}(21 \mathrm{C})$ & 109.5 \\
\hline $\mathrm{C}(12)-\mathrm{C}(22)-\mathrm{H}(22 \mathrm{~A})$ & 109.5 \\
\hline $\mathrm{C}(12)-\mathrm{C}(22)-\mathrm{H}(22 \mathrm{~B})$ & 109.5 \\
\hline
\end{tabular}


$\mathrm{H}(22 \mathrm{~A})-\mathrm{C}(22)-\mathrm{H}(22 \mathrm{~B}) \quad 109.5$

$\mathrm{C}(12)-\mathrm{C}(22)-\mathrm{H}(22 \mathrm{C}) \quad 109.5$

$\mathrm{H}(22 \mathrm{~A})-\mathrm{C}(22)-\mathrm{H}(22 \mathrm{C}) \quad 109.5$

$\mathrm{H}(22 \mathrm{~B})-\mathrm{C}(22)-\mathrm{H}(22 \mathrm{C}) \quad 109.5$ 
Table 10. Torsion angles [deg] for $\mathbf{C}_{\mathbf{1 3}} \mathbf{M} \mathbf{e}_{\mathbf{9}} \mathbf{H}$.

\begin{tabular}{lc}
\hline $\mathrm{C}(13)-\mathrm{C}(1)-\mathrm{C}(2)-\mathrm{C}(3)$ & $172.3(4)$ \\
$\mathrm{C}(14)-\mathrm{C}(1)-\mathrm{C}(2)-\mathrm{C}(3)$ & $-69.4(5)$ \\
$\mathrm{C}(13)-\mathrm{C}(1)-\mathrm{C}(2)-\mathrm{C}(7)$ & $-5.2(4)$ \\
$\mathrm{C}(14)-\mathrm{C}(1)-\mathrm{C}(2)-\mathrm{C}(7)$ & $113.1(4)$ \\
$\mathrm{C}(7)-\mathrm{C}(2)-\mathrm{C}(3)-\mathrm{C}(4)$ & $0.7(6)$ \\
$\mathrm{C}(1)-\mathrm{C}(2)-\mathrm{C}(3)-\mathrm{C}(4)$ & $-176.6(4)$ \\
$\mathrm{C}(7)-\mathrm{C}(2)-\mathrm{C}(3)-\mathrm{C}(15)$ & $179.4(4)$ \\
$\mathrm{C}(1)-\mathrm{C}(2)-\mathrm{C}(3)-\mathrm{C}(15)$ & $2.1(6)$ \\
$\mathrm{C}(2)-\mathrm{C}(3)-\mathrm{C}(4)-\mathrm{C}(5)$ & $3.5(6)$ \\
$\mathrm{C}(15)-\mathrm{C}(3)-\mathrm{C}(4)-\mathrm{C}(5)$ & $-175.3(4)$ \\
$\mathrm{C}(2)-\mathrm{C}(3)-\mathrm{C}(4)-\mathrm{C}(16)$ & $-178.4(4)$ \\
$\mathrm{C}(15)-\mathrm{C}(3)-\mathrm{C}(4)-\mathrm{C}(16)$ & $2.8(6)$ \\
$\mathrm{C}(3)-\mathrm{C}(4)-\mathrm{C}(5)-\mathrm{C}(6)$ & $-1.5(6)$ \\
$\mathrm{C}(16)-\mathrm{C}(4)-\mathrm{C}(5)-\mathrm{C}(6)$ & $-179.6(4)$ \\
$\mathrm{C}(3)-\mathrm{C}(4)-\mathrm{C}(5)-\mathrm{C}(17)$ & $178.2(4)$ \\
$\mathrm{C}(16)-\mathrm{C}(4)-\mathrm{C}(5)-\mathrm{C}(17)$ & $0.1(6)$ \\
$\mathrm{C}(4)-\mathrm{C}(5)-\mathrm{C}(6)-\mathrm{C}(7)$ & $-4.6(6)$ \\
$\mathrm{C}(17)-\mathrm{C}(5)-\mathrm{C}(6)-\mathrm{C}(7)$ & $175.8(4)$ \\
$\mathrm{C}(4)-\mathrm{C}(5)-\mathrm{C}(6)-\mathrm{C}(18)$ & $169.9(4)$ \\
$\mathrm{C}(17)-\mathrm{C}(5)-\mathrm{C}(6)-\mathrm{C}(18)$ & $-9.8(5)$ \\
$\mathrm{C}(5)-\mathrm{C}(6)-\mathrm{C}(7)-\mathrm{C}(2)$ & $8.6(5)$ \\
$\mathrm{C}(18)-\mathrm{C}(6)-\mathrm{C}(7)-\mathrm{C}(2)$ & $-165.6(4)$ \\
$\mathrm{C}(5)-\mathrm{C}(6)-\mathrm{C}(7)-\mathrm{C}(8)$ & $179.9(3)$ \\
$\mathrm{C}(18)-\mathrm{C}(6)-\mathrm{C}(7)-\mathrm{C}(8)$ & $5.8(6)$ \\
$\mathrm{C}(3)-\mathrm{C}(2)-\mathrm{C}(7)-\mathrm{C}(6)$ & $-6.8(6)$ \\
$\mathrm{C}(1)-\mathrm{C}(2)-\mathrm{C}(7)-\mathrm{C}(6)$ & $170.9(3)$ \\
$\mathrm{C}(3)-\mathrm{C}(2)-\mathrm{C}(7)-\mathrm{C}(8)$ & $179.9(3)$ \\
$\mathrm{C}(1)-\mathrm{C}(2)-\mathrm{C}(7)-\mathrm{C}(8)$ & $-2.4(4)$ \\
$\mathrm{C}(6)-\mathrm{C}(7)-\mathrm{C}(8)-\mathrm{C}(9)$ & $22.7(6)$ \\
$\mathrm{C}(2)-\mathrm{C}(7)-\mathrm{C}(8)-\mathrm{C}(9)$ & $-173.3(4)$ \\
$\mathrm{C}(6)-\mathrm{C}(7)-\mathrm{C}(8)-\mathrm{C}(13)$ & $-0.6(6)$ \\
$\mathrm{C}(2)-\mathrm{C}(7)-\mathrm{C}(8)-\mathrm{C}(13)$ & \\
$\mathrm{C}(13)-\mathrm{C}(8)-\mathrm{C}(9)-\mathrm{C}(10)$ & $-165.2(4)$ \\
$\mathrm{C}(7)-\mathrm{C}(8)-\mathrm{C}(9)-\mathrm{C}(10)$ & $-162.2(4)$ \\
$\mathrm{C}(13)-\mathrm{C}(8)-\mathrm{C}(9)-\mathrm{C}(19)$ & $9.8(4)$ \\
$\mathrm{C}(7)-\mathrm{C}(8)-\mathrm{C}(9)-\mathrm{C}(19)$ & $12.6(5)$ \\
$\mathrm{C}(8)-\mathrm{C}(9)-\mathrm{C}(10)-\mathrm{C}(11)$ & $-172.8(4)$ \\
$\mathrm{C}(19)-\mathrm{C}(9)-\mathrm{C}(10)-\mathrm{C}(11)$ & $-159.7(3)$ \\
$\mathrm{C}(8)-\mathrm{C}(9)-\mathrm{C}(10)-\mathrm{C}(20)$ & $14.9(6)$ \\
$\mathrm{C}(19)-\mathrm{C}(9)-\mathrm{C}(10)-\mathrm{C}(20)$ & $-4.4(5)$ \\
$\mathrm{C}(9)-\mathrm{C}(10)-\mathrm{C}(11)-\mathrm{C}(12)$ & $168.3(3)$ \\
$\mathrm{C}(20)-\mathrm{C}(10)-\mathrm{C}(11)-\mathrm{C}(12)$ & $176.1(3)$ \\
$\mathrm{C}(9)-\mathrm{C}(10)-\mathrm{C}(11)-\mathrm{C}(21)$ & $-11.2(5)$ \\
$\mathrm{C}(20)-\mathrm{C}(10)-\mathrm{C}(11)-\mathrm{C}(21)$ & $-5.0(5)$ \\
$\mathrm{C}(10)-\mathrm{C}(11)-\mathrm{C}(12)-\mathrm{C}(13)$ & $174.6(4)$ \\
$\mathrm{C}(21)-\mathrm{C}(11)-\mathrm{C}(12)-\mathrm{C}(13)$ & $-177.8(4)$ \\
$\mathrm{C}(10)-\mathrm{C}(11)-\mathrm{C}(12)-\mathrm{C}(22)$ & \\
$\mathrm{C}(21)-\mathrm{C}(11)-\mathrm{C}(12)-\mathrm{C}(22)$ & \\
&
\end{tabular}


C(11)-C(12)-C(13)-C(8)

$\mathrm{C}(22)-\mathrm{C}(12)-\mathrm{C}(13)-\mathrm{C}(8)$

C(11)-C(12)-C(13)-C(1)

$\mathrm{C}(22)-\mathrm{C}(12)-\mathrm{C}(13)-\mathrm{C}(1)$

$\mathrm{C}(9)-\mathrm{C}(8)-\mathrm{C}(13)-\mathrm{C}(12)$

C(7)-C(8)-C(13)-C(12)

$\mathrm{C}(9)-\mathrm{C}(8)-\mathrm{C}(13)-\mathrm{C}(1)$

$\mathrm{C}(7)-\mathrm{C}(8)-\mathrm{C}(13)-\mathrm{C}(1)$

C(2)-C(1)-C(13)-C(12)

C(14)-C(1)-C(13)-C(12)

$\mathrm{C}(2)-\mathrm{C}(1)-\mathrm{C}(13)-\mathrm{C}(8)$

C(14)-C(1)-C(13)-C(8)
$2.6(6)$

$-178.2(4)$

$-170.9(4)$

$8.4(6)$

$-12.0(5)$

172.1(3)

$162.5(3)$

$-13.4(4)$

$-174.3(4)$

65.7(5)

11.6(4)

$-108.4(4)$ 


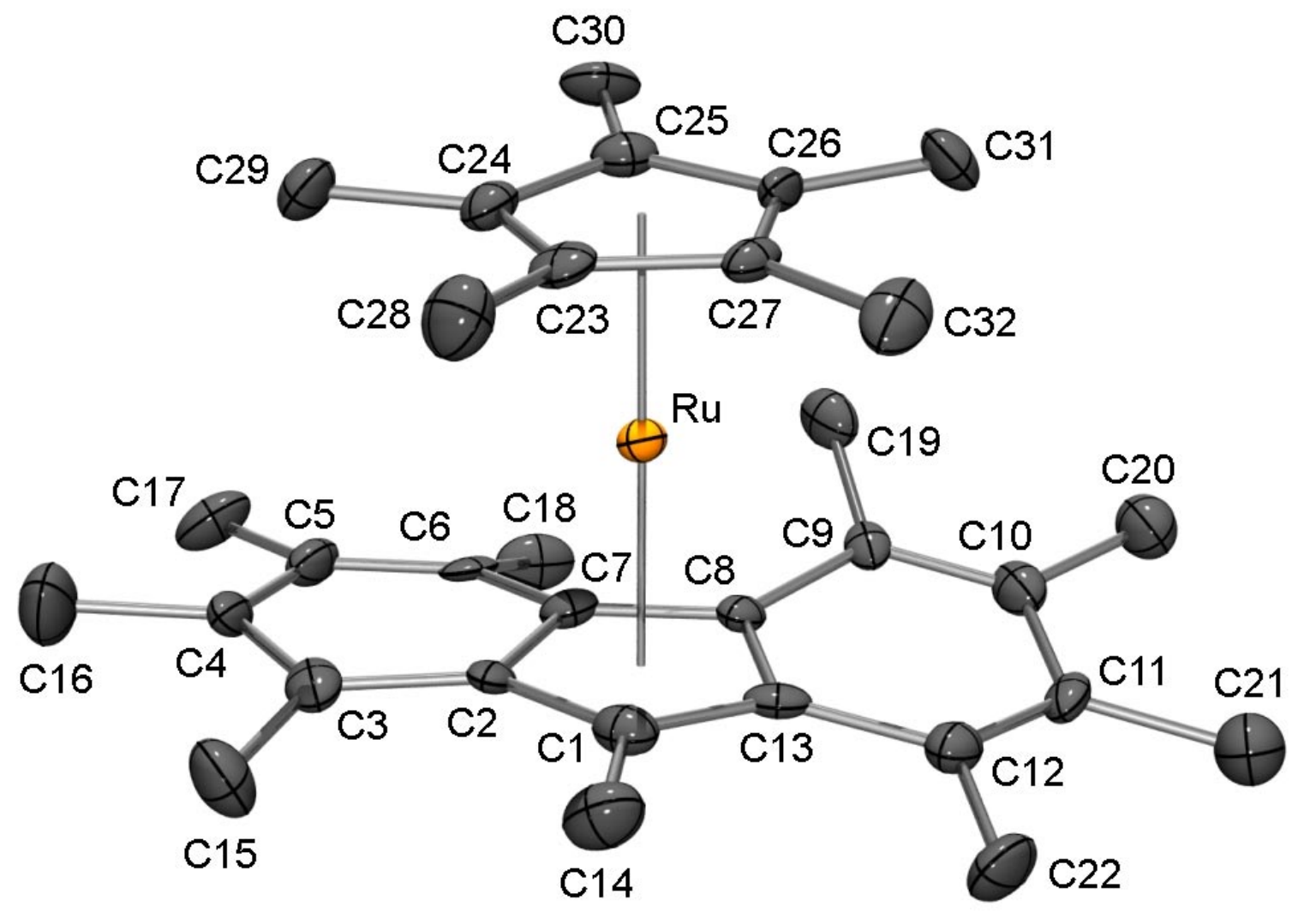

Figure 3. Thermal ellipsoid plot for compound 4. Hydrogen atoms have been omitted for clarity. Thermal ellipsoids are drawn at $50 \%$ probability. 
Empirical Formula

Formula Weight

Temperature

Wavelength

Crystal Color, Habit

Crystal Dimensions

Crystal System

Space Group

Lattice Parameters

Z

Density (calculated)

$\mathrm{F}_{000}$

$\mu(\operatorname{MoK} \alpha)$

Theta range for data collection

Index ranges

Completeness to theta $=23.24^{\circ}$

Reflections collected

Independent reflections

Absorption correction

Max. and min. transmission

Refinement method

Weighting scheme

Data / restraints / parameters

Goodness-of-fit on $\mathrm{F}^{2}$

Final $\mathrm{R}$ indices [I $>2 \operatorname{sigma}(\mathrm{I})]$

$\mathrm{R}$ indices (all data)

Largest diff. peak and hole

\section{G. Crystal and structure refinement for $\mathrm{Cp} *\left(\mathrm{C}_{13} \mathrm{Me}_{9}\right) \mathrm{Ru}$}

$\mathrm{C}_{32} \mathrm{H}_{42} \mathrm{Ru}$

527.73

127(2) K

$0.71073 \AA$

yellow block

$0.3 \times 0.20 \times 0.15 \mathrm{~mm}$

monoclinic

$C \mathrm{c}$

$a=11.262(3) \AA \quad \alpha=90^{\circ}$

$b=31.713(10) \AA$

$c=7.774(2) \AA$

$\mathrm{V}=2546.4(13) \AA^{3}$

$\beta=113.492(4)^{\circ}$

$\gamma=90^{\circ}$
4

$1.377 \mathrm{~g} / \mathrm{cm}^{3}$

1112

$0.634 \mathrm{~mm}^{-1}$

3.13 to $23.24^{\circ}$

$-12<\mathrm{h}<5,-35<\mathrm{k}<35,-8<1<8$

$99.1 \%$

5121

$2454\left(\mathrm{R}_{\mathrm{int}}=0.0312\right)$

semi-empirical from psi-scans

0.996 and 0.982

Full-matrix least squares on $\mathrm{F}^{2}$

$\mathrm{w}=\mathrm{q}\left[\sigma^{2}\left(\mathrm{~F}_{0}^{2}\right)+(\mathrm{aP})^{2}+\mathrm{bP}\right]^{-1}$ where:

$\mathrm{P}=\left(\mathrm{F}_{\mathrm{o}}^{2}+2 \mathrm{~F}_{\mathrm{c}}^{2}\right) / 3, \mathrm{a}=, \mathrm{b}=, \mathrm{q}=1$

2454 / 2 / 298

1.070

$\mathrm{R} 1=0.0343, \mathrm{wR} 2=0.0876$

$\mathrm{R} 1=0.0368, \mathrm{wR} 2=0.0899$

0.452 and $-1.269 \mathrm{e}^{-3}$ 
Table 11. Atomic coordinates [ $\left.\mathrm{x} 10^{4}\right]$ and equivalent isotropic displacement parameters $\left[\AA^{2} \times 10^{3}\right]$ for $\mathrm{Cp} *\left(\mathrm{C}_{13} \mathrm{Me}_{9}\right) \mathrm{Ru}$. $\mathrm{U}(\mathrm{eq})$ is defined as one third of the trace of the orthogonalized $\mathrm{U}_{\mathrm{ij}}$ tensor.

\begin{tabular}{|c|c|c|c|c|}
\hline & $\mathrm{x}$ & $\mathrm{y}$ & $\mathrm{z}$ & $\mathrm{U}(\mathrm{eq})$ \\
\hline $\mathrm{Ru}$ & $3185(1)$ & $6407(1)$ & $6577(1)$ & $16(1)$ \\
\hline $\mathrm{C}(1)$ & $3728(7)$ & $6583(2)$ & $9514(8)$ & $22(2)$ \\
\hline$C(2)$ & $2377(11)$ & $6441(2)$ & $8750(14)$ & 19(2) \\
\hline$C(3)$ & 1198(7) & $6659(2)$ & $8487(9)$ & $26(2)$ \\
\hline C(4) & $47(7)$ & $6451(2)$ & $7574(9)$ & $25(2)$ \\
\hline $\mathrm{C}(5)$ & $20(6)$ & $6009(2)$ & $7150(8)$ & $25(2)$ \\
\hline$C(6)$ & $1127(6)$ & $5784(2)$ & $7542(7)$ & $19(1)$ \\
\hline$C(7)$ & $2355(6)$ & $5997(2)$ & $8191(7)$ & $19(1)$ \\
\hline $\mathrm{C}(8)$ & $3689(6)$ & $5876(2)$ & $8591(7)$ & $17(1)$ \\
\hline $\mathrm{C}(9)$ & $4262(6)$ & $5510(2)$ & $8106(8)$ & $18(1)$ \\
\hline$C(10)$ & $5570(7)$ & $5470(2)$ & $8879(8)$ & $23(1)$ \\
\hline $\mathrm{C}(11)$ & $6412(6)$ & $5792(2)$ & $10100(8)$ & $22(1)$ \\
\hline $\mathrm{C}(12)$ & $5912(6)$ & $6166(2)$ & $10338(8)$ & $23(1)$ \\
\hline$C(13)$ & $4498(7)$ & $6222(2)$ & $9540(9)$ & $20(2)$ \\
\hline $\mathrm{C}(14)$ & $4200(7)$ & $7019(2)$ & $10233(9)$ & $33(2)$ \\
\hline$C(15)$ & $1250(8)$ & $7097(2)$ & $9229(10)$ & $37(2)$ \\
\hline$C(16)$ & $-1209(8)$ & $6678(3)$ & $7114(11)$ & $45(2)$ \\
\hline$C(17)$ & $-1325(7)$ & $5784(2)$ & $6427(10)$ & $38(2)$ \\
\hline $\mathrm{C}(18)$ & $1094(8)$ & $5301(2)$ & $7595(9)$ & $34(2)$ \\
\hline C(19) & $3413(7)$ & $5223(2)$ & $6575(12)$ & $28(2)$ \\
\hline$C(20)$ & $6212(7)$ & $5082(2)$ & $8473(10)$ & $34(2)$ \\
\hline $\mathrm{C}(21)$ & $7856(7)$ & $5702(2)$ & $10978(10)$ & $40(2)$ \\
\hline $\mathrm{C}(22)$ & $6762(7)$ & $6516(2)$ & $11506(10)$ & $35(2)$ \\
\hline $\mathrm{C}(23)$ & $2966(6)$ & $6954(2)$ & $4799(8)$ & $22(1)$ \\
\hline$C(24)$ & $1907(7)$ & $6666(2)$ & 3929(8) & 21(1) \\
\hline$C(25)$ & $2418(8)$ & $6278(3)$ & 3531(9) & $23(2)$ \\
\hline$C(26)$ & $3798(10)$ & $6325(3)$ & $4280(14)$ & $20(2)$ \\
\hline $\mathrm{C}(27)$ & $4144(6)$ & $6736(2)$ & $5072(8)$ & $18(1)$ \\
\hline $\mathrm{C}(28)$ & $2858(7)$ & $7402(2)$ & $5249(9)$ & $33(2)$ \\
\hline C(29) & $498(6)$ & $6753(2)$ & $3374(9)$ & $30(2)$ \\
\hline$C(30)$ & $1617(7)$ & $5907(2)$ & $2522(8)$ & $30(2)$ \\
\hline $\mathrm{C}(31)$ & $4745(7)$ & $5989(2)$ & 4221(9) & $29(2)$ \\
\hline $\mathrm{C}(32)$ & $5481(7)$ & $6903(2)$ & $5954(10)$ & $33(2)$ \\
\hline
\end{tabular}


Table 12. Hydrogen coordinates $\left[\times 10^{4]}\right.$ and isotropic displacement parameters $\left[\AA^{2} \times 10^{3}\right]$.

\begin{tabular}{|c|c|c|c|c|}
\hline & $\mathrm{x}$ & $\mathrm{y}$ & $\mathrm{z}$ & $\mathrm{U}(\mathrm{eq})$ \\
\hline $\mathrm{H}(14 \mathrm{~A})$ & 4398 & 7033 & 11553 & 49 \\
\hline $\mathrm{H}(14 \mathrm{~B})$ & 3539 & 7222 & 9584 & 49 \\
\hline $\mathrm{H}(14 \mathrm{C})$ & 4965 & 7082 & 10021 & 49 \\
\hline $\mathrm{H}(15 \mathrm{~A})$ & 386 & 7196 & 8944 & 56 \\
\hline $\mathrm{H}(15 \mathrm{~B})$ & 1666 & 7280 & 8656 & 56 \\
\hline $\mathrm{H}(15 \mathrm{C})$ & 1730 & 7096 & 10564 & 56 \\
\hline $\mathrm{H}(16 \mathrm{~A})$ & -1040 & 6969 & 7462 & 68 \\
\hline $\mathrm{H}(16 \mathrm{~B})$ & -1668 & 6553 & 7793 & 68 \\
\hline $\mathrm{H}(16 \mathrm{C})$ & -1724 & 6658 & 5792 & 68 \\
\hline $\mathrm{H}(17 \mathrm{~A})$ & -1219 & 5491 & 6204 & 56 \\
\hline $\mathrm{H}(17 \mathrm{~B})$ & -1898 & 5914 & 5281 & 56 \\
\hline $\mathrm{H}(17 \mathrm{C})$ & -1686 & 5808 & 7353 & 56 \\
\hline $\mathrm{H}(18 \mathrm{~A})$ & 211 & 5206 & 7118 & 51 \\
\hline $\mathrm{H}(18 \mathrm{~B})$ & 1537 & 5205 & 8865 & 51 \\
\hline $\mathrm{H}(18 \mathrm{C})$ & 1513 & 5187 & 6836 & 51 \\
\hline $\mathrm{H}(19 \mathrm{~A})$ & 3924 & 4996 & 6413 & 42 \\
\hline $\mathrm{H}(19 \mathrm{~B})$ & 3030 & 5378 & 5425 & 42 \\
\hline $\mathrm{H}(19 \mathrm{C})$ & 2742 & 5110 & 6908 & 42 \\
\hline $\mathrm{H}(20 \mathrm{~A})$ & 5559 & 4892 & 7677 & 50 \\
\hline $\mathrm{H}(20 \mathrm{~B})$ & 6720 & 4942 & 9630 & 50 \\
\hline $\mathrm{H}(20 \mathrm{C})$ & 6763 & 5166 & 7857 & 50 \\
\hline $\mathrm{H}(21 \mathrm{~A})$ & 8302 & 5938 & 11728 & 60 \\
\hline $\mathrm{H}(21 \mathrm{~B})$ & 8163 & 5655 & 10007 & 60 \\
\hline $\mathrm{H}(21 \mathrm{C})$ & 8014 & 5455 & 11755 & 60 \\
\hline $\mathrm{H}(22 \mathrm{~A})$ & 7654 & 6434 & 11921 & 52 \\
\hline $\mathrm{H}(22 \mathrm{~B})$ & 6559 & 6567 & 12575 & 52 \\
\hline $\mathrm{H}(22 \mathrm{C})$ & 6614 & 6768 & 10764 & 52 \\
\hline $\mathrm{H}(28 \mathrm{~A})$ & 2767 & 7574 & 4188 & 49 \\
\hline $\mathrm{H}(28 \mathrm{~B})$ & 3622 & 7484 & 6307 & 49 \\
\hline $\mathrm{H}(28 \mathrm{C})$ & 2113 & 7438 & 5544 & 49 \\
\hline $\mathrm{H}(29 \mathrm{~A})$ & 142 & 6872 & 2136 & 44 \\
\hline $\mathrm{H}(29 B)$ & 391 & 6948 & 4244 & 44 \\
\hline $\mathrm{H}(29 \mathrm{C})$ & 57 & 6495 & 3386 & 44 \\
\hline $\mathrm{H}(30 \mathrm{~A})$ & 1421 & 5930 & 1205 & 45 \\
\hline $\mathrm{H}(30 \mathrm{~B})$ & 827 & 5902 & 2714 & 45 \\
\hline $\mathrm{H}(30 \mathrm{C})$ & 2092 & 5651 & 3001 & 45 \\
\hline $\mathrm{H}(31 \mathrm{~A})$ & 4875 & 6015 & 3078 & 43 \\
\hline $\mathrm{H}(31 \mathrm{~B})$ & 4399 & 5714 & 4274 & 43 \\
\hline $\mathrm{H}(31 \mathrm{C})$ & 5557 & 6024 & 5273 & 43 \\
\hline $\mathrm{H}(32 \mathrm{~A})$ & 5699 & 7042 & 5021 & 50 \\
\hline $\mathrm{H}(32 \mathrm{~B})$ & 6073 & 6675 & 6493 & 50 \\
\hline $\mathrm{H}(32 \mathrm{C})$ & 5538 & 7101 & 6919 & 50 \\
\hline
\end{tabular}


Table 13. Anisotropic displacement parameters $\left[\AA^{2} \times 10^{3}\right]$ for $\mathrm{Cp}^{*}\left(\mathrm{C}_{13} \mathrm{Me}_{9}\right) \mathrm{Ru}$. The anisotropic displacement factor exponent takes the form: $-2 \pi^{2}\left[\left(h a^{*}\right)^{2} U_{11}+\ldots+2 h k a * b * U_{12}\right]$

\begin{tabular}{|c|c|c|c|c|c|c|}
\hline & U11 & U22 & U33 & U23 & U13 & U12 \\
\hline$\overline{\mathrm{Ru}}$ & $16(1)$ & $20(1)$ & $11(1)$ & $0(1)$ & $4(1)$ & $0(1)$ \\
\hline $\mathrm{C}(1)$ & $32(5)$ & $20(4)$ & $16(3)$ & $2(3)$ & $11(3)$ & $2(3)$ \\
\hline $\mathrm{C}(2)$ & $17(5)$ & $34(5)$ & $8(4)$ & $1(3)$ & $7(4)$ & $3(3)$ \\
\hline $\mathrm{C}(3)$ & $30(4)$ & $34(4)$ & $18(3)$ & $13(3)$ & $13(3)$ & $12(3)$ \\
\hline $\mathrm{C}(4)$ & $15(4)$ & $47(4)$ & $14(3)$ & $1(3)$ & $7(3)$ & $7(3)$ \\
\hline$C(5)$ & $15(3)$ & $43(4)$ & $15(3)$ & $3(3)$ & $3(3)$ & $0(3)$ \\
\hline $\mathrm{C}(6)$ & $22(4)$ & $29(3)$ & $4(3)$ & $2(2)$ & $3(3)$ & $-4(3)$ \\
\hline$C(7)$ & $23(4)$ & $23(3)$ & $10(3)$ & $3(2)$ & $4(3)$ & $-1(3)$ \\
\hline $\mathrm{C}(8)$ & $17(3)$ & $24(3)$ & $10(3)$ & $1(2)$ & $7(3)$ & $-1(3)$ \\
\hline $\mathrm{C}(9)$ & $21(4)$ & $20(3)$ & $17(3)$ & $10(2)$ & $11(3)$ & $6(3)$ \\
\hline$C(10)$ & $29(4)$ & $23(3)$ & $21(3)$ & $8(3)$ & $13(3)$ & $9(3)$ \\
\hline $\mathrm{C}(11)$ & $15(3)$ & $36(4)$ & $14(3)$ & $11(3)$ & $4(3)$ & $6(3)$ \\
\hline$C(12)$ & $21(4)$ & $33(4)$ & $16(3)$ & $2(3)$ & $8(3)$ & $-1(3)$ \\
\hline$C(13)$ & $30(5)$ & $22(3)$ & $9(3)$ & $1(3)$ & $9(3)$ & $-3(3)$ \\
\hline $\mathrm{C}(14)$ & $37(4)$ & $31(4)$ & $24(3)$ & $-3(3)$ & $6(3)$ & $-2(3)$ \\
\hline$C(15)$ & $40(5)$ & $41(4)$ & $39(4)$ & $-4(3)$ & $24(4)$ & $9(4)$ \\
\hline$C(16)$ & $27(4)$ & $59(5)$ & $43(4)$ & $-9(4)$ & $7(3)$ & $12(4)$ \\
\hline$C(17)$ & $33(4)$ & $43(4)$ & $22(3)$ & $4(3)$ & $-3(3)$ & $-4(4)$ \\
\hline $\mathrm{C}(18)$ & $40(4)$ & $36(4)$ & $25(4)$ & $-3(3)$ & $12(3)$ & $-18(3)$ \\
\hline C(19) & $37(6)$ & $23(3)$ & $29(3)$ & $5(3)$ & $18(4)$ & $9(3)$ \\
\hline $\mathrm{C}(20)$ & $35(4)$ & $37(4)$ & $31(4)$ & $5(3)$ & $16(3)$ & $8(3)$ \\
\hline $\mathrm{C}(21)$ & $34(5)$ & $54(4)$ & $32(4)$ & $1(3)$ & $13(4)$ & $4(4)$ \\
\hline $\mathrm{C}(22)$ & $20(4)$ & $53(4)$ & $22(4)$ & $-3(3)$ & $-1(3)$ & $-5(3)$ \\
\hline $\mathrm{C}(23)$ & $28(4)$ & $24(3)$ & $12(3)$ & $9(2)$ & $6(3)$ & $3(3)$ \\
\hline $\mathrm{C}(24)$ & $19(4)$ & $29(4)$ & $14(3)$ & $6(3)$ & $4(3)$ & $1(3)$ \\
\hline $\mathrm{C}(25)$ & $29(5)$ & $27(4)$ & $14(4)$ & $4(3)$ & $9(3)$ & $-2(4)$ \\
\hline$C(26)$ & $11(5)$ & $37(4)$ & $11(4)$ & $2(3)$ & $3(3)$ & 1(4) \\
\hline $\mathrm{C}(27)$ & $16(3)$ & $26(3)$ & $11(3)$ & $5(3)$ & $4(3)$ & $-4(3)$ \\
\hline $\mathrm{C}(28)$ & $32(4)$ & $30(3)$ & $34(4)$ & $5(3)$ & 11(3) & $4(3)$ \\
\hline C(29) & $19(4)$ & $44(4)$ & $21(3)$ & $7(3)$ & $3(3)$ & $6(3)$ \\
\hline$C(30)$ & $40(4)$ & $36(4)$ & $13(3)$ & $-7(3)$ & $9(3)$ & $-11(3)$ \\
\hline $\mathrm{C}(31)$ & $31(4)$ & $36(4)$ & $28(3)$ & $0(3)$ & 21(3) & $6(3)$ \\
\hline$C(32)$ & $26(4)$ & $43(4)$ & $31(4)$ & $19(3)$ & $13(3)$ & $-1(3)$ \\
\hline
\end{tabular}


Table 14. Bond lengths $[\AA]$ and angles [deg] for $\mathrm{Cp}^{*}\left(\mathrm{C}_{13} \mathrm{Me}_{9}\right) \mathrm{Ru}$.

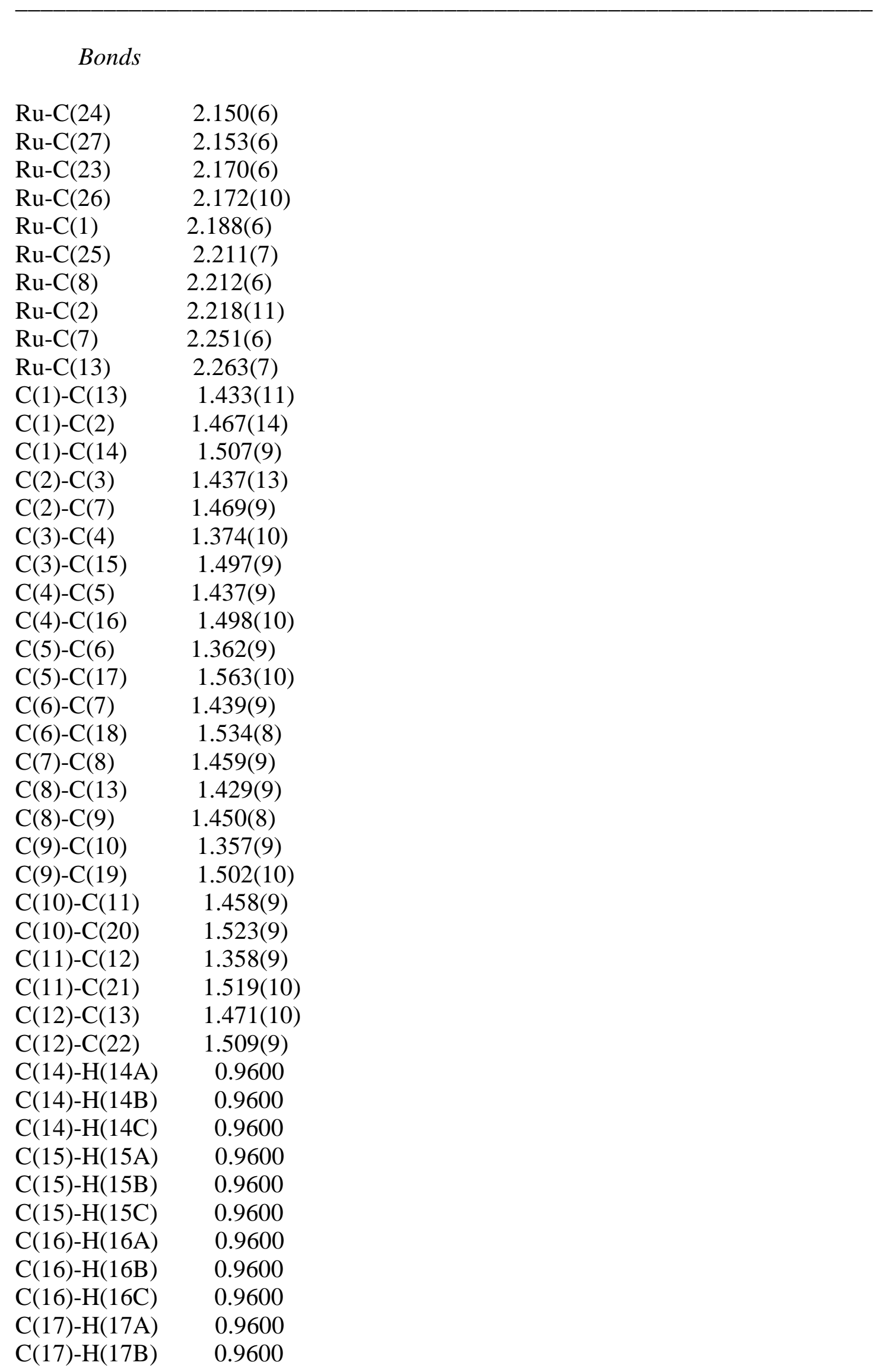




$\begin{array}{ll}\mathrm{C}(17)-\mathrm{H}(17 \mathrm{C}) & 0.9600 \\ \mathrm{C}(18)-\mathrm{H}(18 \mathrm{~A}) & 0.9600 \\ \mathrm{C}(18)-\mathrm{H}(18 \mathrm{~B}) & 0.9600 \\ \mathrm{C}(18)-\mathrm{H}(18 \mathrm{C}) & 0.9600 \\ \mathrm{C}(19)-\mathrm{H}(19 \mathrm{~A}) & 0.9600 \\ \mathrm{C}(19)-\mathrm{H}(19 \mathrm{~B}) & 0.9600 \\ \mathrm{C}(19)-\mathrm{H}(19 \mathrm{C}) & 0.9600 \\ \mathrm{C}(20)-\mathrm{H}(20 \mathrm{~A}) & 0.9600 \\ \mathrm{C}(20)-\mathrm{H}(20 \mathrm{~B}) & 0.9600 \\ \mathrm{C}(20)-\mathrm{H}(20 \mathrm{C}) & 0.9600 \\ \mathrm{C}(21)-\mathrm{H}(21 \mathrm{~A}) & 0.9600 \\ \mathrm{C}(21)-\mathrm{H}(21 \mathrm{~B}) & 0.9600 \\ \mathrm{C}(21)-\mathrm{H}(21 \mathrm{C}) & 0.9600 \\ \mathrm{C}(22)-\mathrm{H}(22 \mathrm{~A}) & 0.9600 \\ \mathrm{C}(22)-\mathrm{H}(22 \mathrm{~B}) & 0.9600 \\ \mathrm{C}(22)-\mathrm{H}(22 \mathrm{C}) & 0.9600 \\ \mathrm{C}(23)-\mathrm{C}(27) & 1.434(9) \\ \mathrm{C}(23)-\mathrm{C}(24) & 1.439(9) \\ \mathrm{C}(23)-\mathrm{C}(28) & 1.480(9) \\ \mathrm{C}(24)-\mathrm{C}(25) & 1.443(11) \\ \mathrm{C}(24)-\mathrm{C}(29) & 1.495(9) \\ \mathrm{C}(25)-\mathrm{C}(26) & 1.434(14) \\ \mathrm{C}(25)-\mathrm{C}(30) & 1.500(10) \\ \mathrm{C}(26)-\mathrm{C}(27) & 1.427(9) \\ \mathrm{C}(26)-\mathrm{C}(31) & 1.522(12) \\ \mathrm{C}(27)-\mathrm{C}(32) & 1.481(10) \\ \mathrm{C}(28)-\mathrm{H}(28 \mathrm{~A}) & 0.9600 \\ \mathrm{C}(28)-\mathrm{H}(28 \mathrm{~B}) & 0.9600 \\ \mathrm{C}(28)-\mathrm{H}(28 \mathrm{C}) & 0.9600 \\ \mathrm{C}(29)-\mathrm{H}(29 \mathrm{~A}) & 0.9600 \\ \mathrm{C}(29)-\mathrm{H}(29 \mathrm{~B}) & 0.9600 \\ \mathrm{C}(29)-\mathrm{H}(29 \mathrm{C}) & 0.9600 \\ \mathrm{C}(30)-\mathrm{H}(30 \mathrm{~A}) & 0.9600 \\ \mathrm{C}(30)-\mathrm{H}(30 \mathrm{~B}) & 0.9600 \\ \mathrm{C}(30)-\mathrm{H}(30 \mathrm{C}) & 0.9600 \\ \mathrm{C}(31)-\mathrm{H}(31 \mathrm{~A}) & 0.9600 \\ \mathrm{C}(31)-\mathrm{H}(31 \mathrm{~B}) & 0.9600 \\ \mathrm{C}(31)-\mathrm{H}(31 \mathrm{C}) & 0.9600 \\ \mathrm{C}(32)-\mathrm{H}(32 \mathrm{~A}) & 0.9600 \\ \mathrm{C}(32)-\mathrm{H}(32 \mathrm{~B}) & 0.9600 \\ \mathrm{C}(32)-\mathrm{H}(32 \mathrm{C}) & 0.9600 \\ & \end{array}$


Angles

$\begin{array}{lc}\mathrm{C}(24)-\mathrm{Ru}-\mathrm{C}(27) & 65.4(3) \\ \mathrm{C}(24)-\mathrm{Ru}-\mathrm{C}(23) & 38.9(2) \\ \mathrm{C}(27)-\mathrm{Ru}-\mathrm{C}(23) & 38.8(2) \\ \mathrm{C}(24)-\mathrm{Ru}-\mathrm{C}(26) & 64.5(3) \\ \mathrm{C}(27)-\mathrm{Ru}-\mathrm{C}(26) & 38.5(2) \\ \mathrm{C}(23)-\mathrm{Ru}-\mathrm{C}(26) & 64.1(3) \\ \mathrm{C}(24)-\mathrm{Ru}-\mathrm{C}(1) & 134.7(3) \\ \mathrm{C}(27)-\mathrm{Ru}-\mathrm{C}(1) & 116.8(2) \\ \mathrm{C}(23)-\mathrm{Ru}-\mathrm{C}(1) & 112.0(2) \\ \mathrm{C}(26)-\mathrm{Ru}-\mathrm{C}(1) & 147.2(3) \\ \mathrm{C}(24)-\mathrm{Ru}-\mathrm{C}(25) & 38.6(3) \\ \mathrm{C}(27)-\mathrm{Ru}-\mathrm{C}(25) & 64.8(3) \\ \mathrm{C}(23)-\mathrm{Ru}-\mathrm{C}(25) & 64.5(3) \\ \mathrm{C}(26)-\mathrm{Ru}-\mathrm{C}(25) & 38.2(4) \\ \mathrm{C}(1)-\mathrm{Ru}-\mathrm{C}(25) & 172.8(3) \\ \mathrm{C}(24)-\mathrm{Ru}-\mathrm{C}(8) & 148.5(2) \\ \mathrm{C}(27)-\mathrm{Ru}-\mathrm{C}(8) & 135.3(2) \\ \mathrm{C}(23)-\mathrm{Ru}-\mathrm{C}(8) & 172.1(2) \\ \mathrm{C}(26)-\mathrm{Ru}-\mathrm{C}(8) & 114.5(3) \\ \mathrm{C}(1)-\mathrm{Ru}-\mathrm{C}(8) & 64.5(2) \\ \mathrm{C}(25)-\mathrm{Ru}-\mathrm{C}(8) & 119.8(3) \\ \mathrm{C}(24)-\mathrm{Ru}-\mathrm{C}(2) & 112.9(3) \\ \mathrm{C}(27)-\mathrm{Ru}-\mathrm{C}(2) & 146.9(3) \\ \mathrm{C}(23)-\mathrm{Ru}-\mathrm{C}(2) & 118.0(3) \\ \mathrm{C}(26)-\mathrm{Ru}-\mathrm{C}(2) & 173.5(5) \\ \mathrm{C}(1)-\mathrm{Ru}-\mathrm{C}(2) & 38.9(4) \\ \mathrm{C}(25)-\mathrm{Ru}-\mathrm{C}(2) & 136.1(4) \\ \mathrm{C}(8)-\mathrm{Ru}-\mathrm{C}(2) & 64.2(3) \\ \mathrm{C}(24)-\mathrm{Ru}-\mathrm{C}(7) & 119.1(2) \\ \mathrm{C}(27)-\mathrm{Ru}-\mathrm{C}(7) & 172.9(2) \\ \mathrm{C}(23)-\mathrm{Ru}-\mathrm{C}(7) & 148.1(2) \\ \mathrm{C}(26)-\mathrm{Ru}-\mathrm{C}(7) & 136.8(3) \\ \mathrm{C}(1)-\mathrm{Ru}-\mathrm{C}(7) & 64.6(2) \\ \mathrm{C}(25)-\mathrm{Ru}-\mathrm{C}(7) & 114.7(3) \\ \mathrm{C}(8)-\mathrm{Ru}-\mathrm{C}(7) & 38.1(2) \\ \mathrm{C}(2)-\mathrm{Ru}-\mathrm{C}(7) & 38.4(2) \\ \mathrm{C}(24)-\mathrm{Ru}-\mathrm{C}(13) & 171.9(3) \\ \mathrm{C}(27)-\mathrm{Ru}-\mathrm{C}(13) & 114.4(2) \\ \mathrm{C}(23)-\mathrm{Ru}-\mathrm{C}(13) & 135.9(2) \\ \mathrm{C}(26)-\mathrm{Ru}-\mathrm{C}(13) & 120.9(3) \\ \mathrm{C}(1)-\mathrm{Ru}-\mathrm{C}(13) & 37.5(3) \\ \mathrm{C}(13)-\mathrm{Ru}(\mathrm{C}) \mathrm{C}) \mathrm{C}(13) & 149.3(2) \\ \mathrm{C}(13)-\mathrm{C}(1)-\mathrm{C}(14) & 127.3(7) \\ & \end{array}$




$\begin{array}{lc}\mathrm{C}(2)-\mathrm{C}(1)-\mathrm{C}(14) & 126.1(6) \\ \mathrm{C}(13)-\mathrm{C}(1)-\mathrm{Ru} & 74.1(3) \\ \mathrm{C}(2)-\mathrm{C}(1)-\mathrm{Ru} & 71.7(5) \\ \mathrm{C}(14)-\mathrm{C}(1)-\mathrm{Ru} & 122.2(5) \\ \mathrm{C}(3)-\mathrm{C}(2)-\mathrm{C}(1) & 131.2(6) \\ \mathrm{C}(3)-\mathrm{C}(2)-\mathrm{C}(7) & 120.9(8) \\ \mathrm{C}(1)-\mathrm{C}(2)-\mathrm{C}(7) & 107.9(8) \\ \mathrm{C}(3)-\mathrm{C}(2)-\mathrm{Ru} & 123.4(6) \\ \mathrm{C}(1)-\mathrm{C}(2)-\mathrm{Ru} & 69.5(5) \\ \mathrm{C}(7)-\mathrm{C}(2)-\mathrm{Ru} & 72.0(5) \\ \mathrm{C}(4)-\mathrm{C}(3)-\mathrm{C}(2) & 118.1(6) \\ \mathrm{C}(4)-\mathrm{C}(3)-\mathrm{C}(15) & 121.9(6) \\ \mathrm{C}(2)-\mathrm{C}(3)-\mathrm{C}(15) & 120.0(7) \\ \mathrm{C}(3)-\mathrm{C}(4)-\mathrm{C}(5) & 121.0(6) \\ \mathrm{C}(3)-\mathrm{C}(4)-\mathrm{C}(16) & 119.9(6) \\ \mathrm{C}(5)-\mathrm{C}(4)-\mathrm{C}(16) & 118.9(6) \\ \mathrm{C}(6)-\mathrm{C}(5)-\mathrm{C}(4) & 121.8(6) \\ \mathrm{C}(6)-\mathrm{C}(5)-\mathrm{C}(17) & 120.7(6) \\ \mathrm{C}(4)-\mathrm{C}(5)-\mathrm{C}(17) & 117.2(6) \\ \mathrm{C}(5)-\mathrm{C}(6)-\mathrm{C}(7) & 119.9(5) \\ \mathrm{C}(5)-\mathrm{C}(6)-\mathrm{C}(18) & 120.0(6) \\ \mathrm{C}(7)-\mathrm{C}(6)-\mathrm{C}(18) & 119.4(6) \\ \mathrm{C}(6)-\mathrm{C}(7)-\mathrm{C}(8) & 135.6(5) \\ \mathrm{C}(6)-\mathrm{C}(7)-\mathrm{C}(2) & 117.1(7) \\ \mathrm{C}(8)-\mathrm{C}(7)-\mathrm{C}(2) & 107.1(6) \\ \mathrm{C}(6)-\mathrm{C}(7)-\mathrm{Ru} & 129.1(4) \\ \mathrm{C}(8)-\mathrm{C}(7)-\mathrm{Ru} & 69.5(3) \\ \mathrm{C}(2)-\mathrm{C}(7)-\mathrm{Ru} & 69.6(5) \\ \mathrm{C}(13)-\mathrm{C}(8)-\mathrm{C}(9) & 119.4(6) \\ \mathrm{C}(13)-\mathrm{C}(8)-\mathrm{C}(7) & 107.4(5) \\ \mathrm{C}(9)-\mathrm{C}(8)-\mathrm{C}(7) & 133.1(5) \\ \mathrm{C}(13)-\mathrm{C}(8)-\mathrm{Ru} & 73.3(3) \\ \mathrm{C}(9)-\mathrm{C}(8)-\mathrm{Ru} & 116.1(4) \\ \mathrm{C}(7)-\mathrm{C}(8)-\mathrm{Ru} & 72.4(3) \\ \mathrm{C}(10)-\mathrm{C}(9)-\mathrm{C}(8) & 118.6(6) \\ \mathrm{C}(10)-\mathrm{C}(9)-\mathrm{C}(19) & 122.0(6) \\ \mathrm{C}(8)-\mathrm{C}(9)-\mathrm{C}(19) & 118.9(5) \\ \mathrm{C}(9)-\mathrm{C}(10)-\mathrm{C}(11) & 122.1(6) \\ \mathrm{C}(9)-\mathrm{C}(10)-\mathrm{C}(20) & 120.4(6) \\ \mathrm{C}(11)-\mathrm{C}(10)-\mathrm{C}(20) & 117.5(6) \\ \mathrm{C}(12)-\mathrm{C}(11)-\mathrm{C}(10) & 120.3(6) \\ \mathrm{C}(12)-\mathrm{C}(11)-\mathrm{C}(21) & 122.1(6) \\ \mathrm{C}(10)-\mathrm{C}(11)-\mathrm{C}(21) & 117.6(6) \\ \mathrm{C}(11)-\mathrm{C}(12)-\mathrm{C}(13) & 119.1(6) \\ \mathrm{C}(13)-\mathrm{C}(12)-\mathrm{C}(22) & 121.8(6) \\ \mathrm{C}(8)-\mathrm{C}(13)-\mathrm{C}(1) & 110.4(7) \\ \mathrm{C}(8)-\mathrm{C}(13)-\mathrm{C}(12) & 119.1(6) \\ \mathrm{C}(1)-\mathrm{C}(13)-\mathrm{C}(12) & 130.5(7) \\ \mathrm{C}(8)-\mathrm{C}(13)-\mathrm{Ru} & 69.5(3) \\ \mathrm{C}(1)-\mathrm{C}(13)-\mathrm{Ru} & 68.4(3) \\ & \end{array}$




\begin{tabular}{|c|c|}
\hline $\mathrm{C}(12)-\mathrm{C}(13)-\mathrm{Ru}$ & $128.2(4)$ \\
\hline $\mathrm{C}(1)-\mathrm{C}(14)-\mathrm{H}(14 \mathrm{~A})$ & 109.5 \\
\hline $\mathrm{C}(1)-\mathrm{C}(14)-\mathrm{H}(14 \mathrm{~B})$ & 109.5 \\
\hline $\mathrm{H}(14 \mathrm{~A})-\mathrm{C}(14)-\mathrm{H}(14 \mathrm{~B})$ & 109.5 \\
\hline $\mathrm{C}(1)-\mathrm{C}(14)-\mathrm{H}(14 \mathrm{C})$ & 109.5 \\
\hline $\mathrm{H}(14 \mathrm{~A})-\mathrm{C}(14)-\mathrm{H}(14 \mathrm{C})$ & 109.5 \\
\hline $\mathrm{H}(14 \mathrm{~B})-\mathrm{C}(14)-\mathrm{H}(14 \mathrm{C})$ & 109.5 \\
\hline $\mathrm{C}(3)-\mathrm{C}(15)-\mathrm{H}(15 \mathrm{~A})$ & 109.5 \\
\hline $\mathrm{C}(3)-\mathrm{C}(15)-\mathrm{H}(15 \mathrm{~B})$ & 109.5 \\
\hline $\mathrm{H}(15 \mathrm{~A})-\mathrm{C}(15)-\mathrm{H}(15 \mathrm{~B})$ & 109.5 \\
\hline $\mathrm{C}(3)-\mathrm{C}(15)-\mathrm{H}(15 \mathrm{C})$ & 109.5 \\
\hline $\mathrm{H}(15 \mathrm{~A})-\mathrm{C}(15)-\mathrm{H}(15 \mathrm{C})$ & 109.5 \\
\hline $\mathrm{H}(15 \mathrm{~B})-\mathrm{C}(15)-\mathrm{H}(15 \mathrm{C})$ & 109.5 \\
\hline $\mathrm{C}(4)-\mathrm{C}(16)-\mathrm{H}(16 \mathrm{~A})$ & 109.5 \\
\hline $\mathrm{C}(4)-\mathrm{C}(16)-\mathrm{H}(16 \mathrm{~B})$ & 109.5 \\
\hline $\mathrm{H}(16 \mathrm{~A})-\mathrm{C}(16)-\mathrm{H}(16 \mathrm{~B})$ & 109.5 \\
\hline $\mathrm{C}(4)-\mathrm{C}(16)-\mathrm{H}(16 \mathrm{C})$ & 109.5 \\
\hline $\mathrm{H}(16 \mathrm{~A})-\mathrm{C}(16)-\mathrm{H}(16 \mathrm{C})$ & 109.5 \\
\hline $\mathrm{H}(16 \mathrm{~B})-\mathrm{C}(16)-\mathrm{H}(16 \mathrm{C})$ & 109.5 \\
\hline $\mathrm{C}(5)-\mathrm{C}(17)-\mathrm{H}(17 \mathrm{~A})$ & 109.5 \\
\hline $\mathrm{C}(5)-\mathrm{C}(17)-\mathrm{H}(17 \mathrm{~B})$ & 109.5 \\
\hline $\mathrm{H}(17 \mathrm{~A})-\mathrm{C}(17)-\mathrm{H}(17 \mathrm{~B})$ & 109.5 \\
\hline $\mathrm{C}(5)-\mathrm{C}(17)-\mathrm{H}(17 \mathrm{C})$ & 109.5 \\
\hline $\mathrm{H}(17 \mathrm{~A})-\mathrm{C}(17)-\mathrm{H}(17 \mathrm{C})$ & 109.5 \\
\hline $\mathrm{H}(17 \mathrm{~B})-\mathrm{C}(17)-\mathrm{H}(17 \mathrm{C})$ & 109.5 \\
\hline $\mathrm{C}(6)-\mathrm{C}(18)-\mathrm{H}(18 \mathrm{~A})$ & 109.5 \\
\hline $\mathrm{C}(6)-\mathrm{C}(18)-\mathrm{H}(18 \mathrm{~B})$ & 109.5 \\
\hline $\mathrm{H}(18 \mathrm{~A})-\mathrm{C}(18)-\mathrm{H}(18 \mathrm{~B})$ & 109.5 \\
\hline $\mathrm{C}(6)-\mathrm{C}(18)-\mathrm{H}(18 \mathrm{C})$ & 109.5 \\
\hline $\mathrm{H}(18 \mathrm{~A})-\mathrm{C}(18)-\mathrm{H}(18 \mathrm{C})$ & 109.5 \\
\hline $\mathrm{H}(18 \mathrm{~B})-\mathrm{C}(18)-\mathrm{H}(18 \mathrm{C})$ & 109.5 \\
\hline $\mathrm{C}(9)-\mathrm{C}(19)-\mathrm{H}(19 \mathrm{~A})$ & 109.5 \\
\hline $\mathrm{C}(9)-\mathrm{C}(19)-\mathrm{H}(19 \mathrm{~B})$ & 109.5 \\
\hline $\mathrm{H}(19 \mathrm{~A})-\mathrm{C}(19)-\mathrm{H}(19 \mathrm{~B})$ & 109.5 \\
\hline $\mathrm{C}(9)-\mathrm{C}(19)-\mathrm{H}(19 \mathrm{C})$ & 109.5 \\
\hline $\mathrm{H}(19 \mathrm{~A})-\mathrm{C}(19)-\mathrm{H}(19 \mathrm{C})$ & 109.5 \\
\hline $\mathrm{H}(19 \mathrm{~B})-\mathrm{C}(19)-\mathrm{H}(19 \mathrm{C})$ & 109.5 \\
\hline $\mathrm{C}(10)-\mathrm{C}(20)-\mathrm{H}(20 \mathrm{~A})$ & 109.5 \\
\hline $\mathrm{C}(10)-\mathrm{C}(20)-\mathrm{H}(20 \mathrm{~B})$ & 109.5 \\
\hline $\mathrm{H}(20 \mathrm{~A})-\mathrm{C}(20)-\mathrm{H}(20 \mathrm{~B})$ & 109.5 \\
\hline $\mathrm{C}(10)-\mathrm{C}(20)-\mathrm{H}(20 \mathrm{C})$ & 109.5 \\
\hline $\mathrm{H}(20 \mathrm{~A})-\mathrm{C}(20)-\mathrm{H}(20 \mathrm{C})$ & 109.5 \\
\hline $\mathrm{H}(20 \mathrm{~B})-\mathrm{C}(20)-\mathrm{H}(20 \mathrm{C})$ & 109.5 \\
\hline $\mathrm{C}(11)-\mathrm{C}(21)-\mathrm{H}(21 \mathrm{~A})$ & 109.5 \\
\hline $\mathrm{C}(11)-\mathrm{C}(21)-\mathrm{H}(21 \mathrm{~B})$ & 109.5 \\
\hline$H(21 A)-C(21)-H(21 B)$ & 109.5 \\
\hline $\mathrm{C}(11)-\mathrm{C}(21)-\mathrm{H}(21 \mathrm{C})$ & 109.5 \\
\hline $\mathrm{H}(21 \mathrm{~A})-\mathrm{C}(21)-\mathrm{H}(21 \mathrm{C})$ & 109.5 \\
\hline $\mathrm{H}(21 \mathrm{~B})-\mathrm{C}(21)-\mathrm{H}(21 \mathrm{C})$ & 109.5 \\
\hline $\mathrm{C}(12)-\mathrm{C}(22)-\mathrm{H}(22 \mathrm{~A})$ & 109.5 \\
\hline $\mathrm{C}(12)-\mathrm{C}(22)-\mathrm{H}(22 \mathrm{~B})$ & 109.5 \\
\hline
\end{tabular}




\begin{tabular}{|c|c|}
\hline$(22 \mathrm{~A})-\mathrm{C}(22)-\mathrm{H}(22 \mathrm{~B})$ & 109.5 \\
\hline $\mathrm{C}(12)-\mathrm{C}(22)-\mathrm{H}(22 \mathrm{C})$ & 109.5 \\
\hline $\mathrm{H}(22 \mathrm{~A})-\mathrm{C}(22)-\mathrm{H}(22 \mathrm{C})$ & 109.5 \\
\hline $\mathrm{H}(22 \mathrm{~B})-\mathrm{C}(22)-\mathrm{H}(22 \mathrm{C})$ & 109.5 \\
\hline $\mathrm{C}(27)-\mathrm{C}(23)-\mathrm{C}(24)$ & $108.0(5)$ \\
\hline $\mathrm{C}(27)-\mathrm{C}(23)-\mathrm{C}(28)$ & $125.9(6)$ \\
\hline $\mathrm{C}(24)-\mathrm{C}(23)-\mathrm{C}(28)$ & $126.1(6)$ \\
\hline$C(27)-C(23)-R u$ & $70.0(3)$ \\
\hline $\mathrm{C}(24)-\mathrm{C}(23)-\mathrm{Ru}$ & $69.8(3)$ \\
\hline $\mathrm{C}(28)-\mathrm{C}(23)-\mathrm{Ru}$ & $128.0(4)$ \\
\hline$C(23)-C(24)-C(25)$ & $108.4(7)$ \\
\hline $\mathrm{C}(23)-\mathrm{C}(24)-\mathrm{C}(29)$ & $127.1(6)$ \\
\hline$C(25)-C(24)-C(29)$ & $124.3(6)$ \\
\hline $\mathrm{C}(23)-\mathrm{C}(24)-\mathrm{Ru}$ & $71.3(3)$ \\
\hline $\mathrm{C}(25)-\mathrm{C}(24)-\mathrm{Ru}$ & $73.0(3)$ \\
\hline $\mathrm{C}(29)-\mathrm{C}(24)-\mathrm{Ru}$ & $125.1(5)$ \\
\hline$C(26)-C(25)-C(24)$ & $106.5(7)$ \\
\hline$C(26)-C(25)-C(30)$ & $128.6(8)$ \\
\hline$C(24)-C(25)-C(30)$ & $125.0(7)$ \\
\hline$C(26)-C(25)-R u$ & $69.4(5)$ \\
\hline $\mathrm{C}(24)-\mathrm{C}(25)-\mathrm{Ru}$ & $68.4(4)$ \\
\hline $\mathrm{C}(30)-\mathrm{C}(25)-\mathrm{Ru}$ & $127.0(5)$ \\
\hline$C(27)-C(26)-C(25)$ & $109.7(8)$ \\
\hline $\mathrm{C}(27)-\mathrm{C}(26)-\mathrm{C}(31)$ & $125.4(8)$ \\
\hline$C(25)-C(26)-C(31)$ & $124.9(8)$ \\
\hline $\mathrm{C}(27)-\mathrm{C}(26)-\mathrm{Ru}$ & $70.0(5)$ \\
\hline$C(25)-C(26)-R u$ & $72.4(6)$ \\
\hline $\mathrm{C}(31)-\mathrm{C}(26)-\mathrm{Ru}$ & $124.4(6)$ \\
\hline$C(26)-C(27)-C(23)$ & $107.3(7)$ \\
\hline$C(26)-C(27)-C(32)$ & $125.3(7)$ \\
\hline$C(23)-C(27)-C(32)$ & $127.3(6)$ \\
\hline$C(26)-C(27)-R u$ & $71.5(5)$ \\
\hline $\mathrm{C}(23)-\mathrm{C}(27)-\mathrm{Ru}$ & $71.3(3)$ \\
\hline $\mathrm{C}(32)-\mathrm{C}(27)-\mathrm{Ru}$ & $124.0(4)$ \\
\hline $\mathrm{C}(23)-\mathrm{C}(28)-\mathrm{H}(28 \mathrm{~A})$ & 109.5 \\
\hline $\mathrm{C}(23)-\mathrm{C}(28)-\mathrm{H}(28 \mathrm{~B})$ & 109.5 \\
\hline $\mathrm{H}(28 \mathrm{~A})-\mathrm{C}(28)-\mathrm{H}(28 \mathrm{~B})$ & 109.5 \\
\hline $\mathrm{C}(23)-\mathrm{C}(28)-\mathrm{H}(28 \mathrm{C})$ & 109.5 \\
\hline $\mathrm{H}(28 \mathrm{~A})-\mathrm{C}(28)-\mathrm{H}(28 \mathrm{C})$ & 109.5 \\
\hline $\mathrm{H}(28 \mathrm{~B})-\mathrm{C}(28)-\mathrm{H}(28 \mathrm{C})$ & 109.5 \\
\hline $\mathrm{C}(24)-\mathrm{C}(29)-\mathrm{H}(29 \mathrm{~A})$ & 109.5 \\
\hline $\mathrm{C}(24)-\mathrm{C}(29)-\mathrm{H}(29 \mathrm{~B})$ & 109.5 \\
\hline $\mathrm{H}(29 \mathrm{~A})-\mathrm{C}(29)-\mathrm{H}(29 \mathrm{~B})$ & 109.5 \\
\hline $\mathrm{C}(24)-\mathrm{C}(29)-\mathrm{H}(29 \mathrm{C})$ & 109.5 \\
\hline $\mathrm{H}(29 \mathrm{~A})-\mathrm{C}(29)-\mathrm{H}(29 \mathrm{C})$ & 109.5 \\
\hline $\mathrm{H}(29 \mathrm{~B})-\mathrm{C}(29)-\mathrm{H}(29 \mathrm{C})$ & 109.5 \\
\hline $\mathrm{C}(25)-\mathrm{C}(30)-\mathrm{H}(30 \mathrm{~A})$ & 109.5 \\
\hline $\mathrm{C}(25)-\mathrm{C}(30)-\mathrm{H}(30 \mathrm{~B})$ & 109.5 \\
\hline $\mathrm{H}(30 \mathrm{~A})-\mathrm{C}(30)-\mathrm{H}(30 \mathrm{~B})$ & 109.5 \\
\hline $\mathrm{C}(25)-\mathrm{C}(30)-\mathrm{H}(30 \mathrm{C})$ & 109.5 \\
\hline $\mathrm{H}(30 \mathrm{~A})-\mathrm{C}(30)-\mathrm{H}(30 \mathrm{C})$ & 109.5 \\
\hline
\end{tabular}




\begin{tabular}{lc}
$\mathrm{H}(30 \mathrm{~B})-\mathrm{C}(30)-\mathrm{H}(30 \mathrm{C})$ & 109.5 \\
$\mathrm{C}(26)-\mathrm{C}(31)-\mathrm{H}(31 \mathrm{~A})$ & 109.5 \\
$\mathrm{C}(26)-\mathrm{C}(31)-\mathrm{H}(31 \mathrm{~B})$ & 109.5 \\
$\mathrm{H}(31 \mathrm{~A})-\mathrm{C}(31)-\mathrm{H}(31 \mathrm{~B})$ & 109.5 \\
$\mathrm{C}(26)-\mathrm{C}(31)-\mathrm{H}(31 \mathrm{C})$ & 109.5 \\
$\mathrm{H}(31 \mathrm{~A})-\mathrm{C}(31)-\mathrm{H}(31 \mathrm{C})$ & 109.5 \\
$\mathrm{H}(31 \mathrm{~B})-\mathrm{C}(31)-\mathrm{H}(31 \mathrm{C})$ & 109.5 \\
$\mathrm{C}(27)-\mathrm{C}(32)-\mathrm{H}(32 \mathrm{~A})$ & 109.5 \\
$\mathrm{C}(27)-\mathrm{C}(32)-\mathrm{H}(32 \mathrm{~B})$ & 109.5 \\
$\mathrm{H}(32 \mathrm{~A})-\mathrm{C}(32)-\mathrm{H}(32 \mathrm{~B})$ & 109.5 \\
$\mathrm{C}(27)-\mathrm{C}(32)-\mathrm{H}(32 \mathrm{C})$ & 109.5 \\
$\mathrm{H}(32 \mathrm{~A})-\mathrm{C}(32)-\mathrm{H}(32 \mathrm{C})$ & 109.5 \\
$\mathrm{H}(32 \mathrm{~B})-\mathrm{C}(32)-\mathrm{H}(32 \mathrm{C})$ & 109.5 \\
\hline
\end{tabular}

Table 15. Torsion angles [deg] for $\mathrm{Cp}^{*}\left(\mathrm{C}_{13} \mathrm{Me}_{9}\right) \mathrm{Ru}$.

$\mathrm{C}(6)-\mathrm{C}(7)-\mathrm{C}(8)-\mathrm{C}(9)$

